\title{
Different Reaction Modes for the Oxidative Dimerization of Epoxyquinols and Epoxyquinones - Importance of the Intermolecular Hydrogen-Bonding
}

Mitsuru Shoji, ${ }^{a}$ Hiroki Imai, ${ }^{\mathrm{a}}$ Isamu Shiina, ${ }^{\mathrm{b}}$ Hideaki Kakeya, ${ }^{\mathrm{c}}$ Hiroyuki Osada, ${ }^{\mathrm{c}}$ Yujiro Hayashi ${ }^{\mathrm{a}, *}$

${ }^{a}$ Department of Industrial Chemistry, Faculty of Engineering, Tokyo University of Science, Kagurazaka, Shinjuku-ku, Tokyo 162-8601, Japan

${ }^{\mathrm{b}}$ Department of Applied Chemistry, Faculty of Science, Tokyo University of Science, Kagurazaka, Shinjuku-ku, Tokyo 162-8601, Japan

'Antibiotics Laboratory, Discovery Research Institute, RIKEN, 2-1 Hirosawa, Wako, Saitama 3510198, Japan

\section{Table of contents}

S2 - S6: Synthetic procedures of new compounds and characterization of all new compounds S7 - S 21: Cartesian Coordinates 


\section{Experimental Section}<smiles>C/C=C/C1=C(C[OH2+])[C@H](O)[C@H]2OC2C1=O</smiles>

(1R, 5S, 6R)-4-(tert-Butyldimethylsiloxymethyl)-5-hydroxy-3-propenyl7-oxabicyclo[4.1.0]hept-3-en-2-one (12). To a solution of 3 (22.6 mg, $0.115 \mathrm{mmol}), \mathrm{Et}_{3} \mathrm{~N}$ (48 ml, $\left.0.346 \mathrm{mmol}\right)$ and DMAP (1.7 mg, $\left.0.014 \mathrm{mmol}\right)$ in dry $\mathrm{CH}_{2} \mathrm{Cl}_{2}(0.75 \mathrm{ml})$ was added $\mathrm{TBSCl}(34.7 \mathrm{mg}, 0.230 \mathrm{mmol})$ at $0{ }^{\circ} \mathrm{C}$ and stirred for $4.5 \mathrm{~h}$ under argon atmosphere. The reaction mixture was quenched with saturated aqueous $\mathrm{NH}_{4} \mathrm{Cl}$ and diluted with AcOEt. The organic phase was washed with saturated aqueous $\mathrm{NaCl}$ and dried over anhydrous $\mathrm{Na}_{2} \mathrm{SO}_{4}$, then concentrated under reduced pressure. The residue was purified by silica gel column chromatography (AcOEt/Hexane=1/5 1/3) to afford $12(28.6 \mathrm{mg}$, $80 \%)$ as a colorless oil. ${ }^{1} \mathrm{H}$ NMR $\left(400 \mathrm{MHz} \mathrm{CDCl}_{3}\right) \delta 0.13(3 \mathrm{H}, \mathrm{s}), 0.14(1 \mathrm{H}, \mathrm{s}), 0.93(9 \mathrm{H}, \mathrm{s}), 1.83$ $(3 \mathrm{H}, \mathrm{dd}, J=6.4,1.3 \mathrm{~Hz}), 3.52(1 \mathrm{H}, \mathrm{br}-\mathrm{s}), 3.53(1 \mathrm{H}, \mathrm{d}, J=3.7 \mathrm{~Hz}), 3.79(1 \mathrm{H}, \mathrm{dd}, J=3.7,1.5 \mathrm{~Hz}), 4.12$ $(1 \mathrm{H}, \mathrm{d}, J=14.5 \mathrm{~Hz}), 4.72(1 \mathrm{H}, \mathrm{d}, J=14.5 \mathrm{~Hz}), 4.91(1 \mathrm{H}, \mathrm{br}-\mathrm{s}), 5.89(1 \mathrm{H}, \mathrm{dq}, J=16.0,6.4 \mathrm{~Hz}), 6.02(1 \mathrm{H}$, br-d, $J=16.0 \mathrm{~Hz}) ;{ }^{13} \mathrm{C}$ NMR $\left(100 \mathrm{MHz} \mathrm{CDCl}_{3}\right) \delta-5.62,18.10,19.08,25.75,53.37,55.45,63.68$, 65.00, 121.61, 130.00, 134.79, 147.05, 194.15; FT-IR (neat) v 3460, 2954, 2929, 2858, 1684, 1255, 1080, 837, $779 \mathrm{~cm}^{-1}$; HRMS (FAB) $[\mathrm{M}+\mathrm{H}]^{+}$calculated for $\mathrm{C}_{16} \mathrm{H}_{27} \mathrm{O}_{4} \mathrm{Si}$ : 311.1679, found: 311.1655 ; $[\alpha]_{\mathrm{D}}^{19}=+160(c=0.48, \mathrm{MeOH})$.

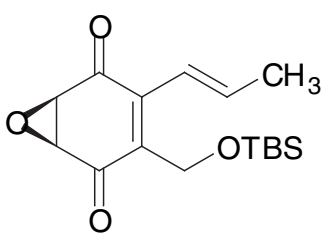

13

(1S, 6R)-3-(tert-Butyldimethylsiloxymethyl)-4-propenyl-7-oxabicyclo[4.1.0]hept-3-ene-2,5-dione (13). To a solution of 12 (28.6 mg, 0.0921 $\mathrm{mmol})$ in dry $\mathrm{CH}_{2} \mathrm{Cl}_{2}(0.5 \mathrm{ml})$ was added Dess-Martin periodinane (58.6 $\mathrm{mg}, 0.138 \mathrm{mmol})$ at $0{ }^{\circ} \mathrm{C}$ under argon atmosphere and stirred for $10 \mathrm{~min}$ at room temperature. The reaction mixture was quenched with saturated aqueous $\mathrm{NaHCO}_{3}$ and diluted with AcOEt. The organic phase was washed with saturated aqueous $\mathrm{NaHCO}_{3}$ and saturated aqueous $\mathrm{NaCl}$, and dried over anhydrous $\mathrm{Na}_{2} \mathrm{SO}_{4}$, then concentrated under reduced pressure. The residue was purified by silica gel column chromatography (AcOEt/Hexane=0/1 1/10) to afford 13 (25.6 mg, 90\%) as a colorless oil. ${ }^{1} \mathrm{H} \mathrm{NMR}\left(400 \mathrm{MHz}, \mathrm{CDCl}_{3}\right) \delta 0.08(6 \mathrm{H}, \mathrm{s}), 0.88(9 \mathrm{H}, \mathrm{s}), 1.93(3 \mathrm{H}$, $\mathrm{dd}, J=6.8,1.6 \mathrm{~Hz}), 3.84(1 \mathrm{H}, \mathrm{d}, J=4.1 \mathrm{~Hz}), 3.86(1 \mathrm{H}, \mathrm{d}, J=4.1 \mathrm{~Hz}), 4.37(1 \mathrm{H}, \mathrm{d}, J=11.6 \mathrm{~Hz}), 4.60(1 \mathrm{H}$, d, $J=11.6 \mathrm{~Hz}), 6.48(1 \mathrm{H}, \mathrm{dq}, J=15.8,1.6 \mathrm{~Hz}), 6.70(1 \mathrm{H}, \mathrm{dq}, J=15.8,6.8 \mathrm{~Hz}) ;{ }^{13} \mathrm{C} \mathrm{NMR}(100 \mathrm{MHz}$, $\left.\mathrm{CDCl}_{3}\right) \delta-5.37,-5.29,18.20,20.08,25.75,53.67,53.96,56.44,122.79,137.14,141.30,141.72$, 192.13, 193.62; FT-IR (neat) n 2956, 2929, 2858, 1697, 1685, 1629, 1259, 1086, $837 \mathrm{~cm}^{-1}$; HRMS $(\mathrm{FAB})[\mathrm{M}+\mathrm{H}]^{+}$calculated for $\mathrm{C}_{16} \mathrm{H}_{25} \mathrm{O}_{4} \mathrm{Si}: 309.1522$, found: $309.1519 ;[\alpha]_{\mathrm{D}}{ }^{20}=+23.9(c=0.77, \mathrm{MeOH})$.

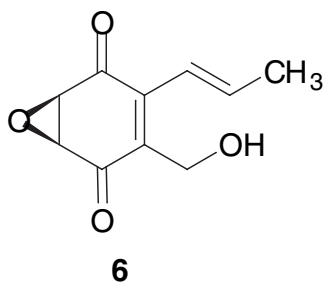

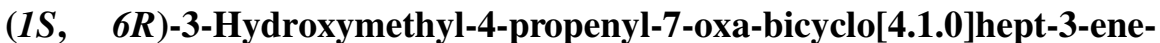
2,5-dione (6). ${ }^{1}$ To a solution of $13(25.6 \mathrm{mg}, 0.0829 \mathrm{mmol})$ in $\mathrm{MeOH}(0.5$ $\mathrm{ml})$ was added Dowex ${ }^{\circledR} 50 \mathrm{~W}-\mathrm{X} 4(12.8 \mathrm{mg})$ at room temperature and stirred for $11 \mathrm{~h}$. The reaction mixture was filtered and concentrated under reduced pressure. The residue was purified by preparative thin-layer chromatography (AcOEt/Hexane $=1 / 3)$ to afford $6(13.1 \mathrm{mg}, 81 \%)$ as an yellow oil. ${ }^{1} \mathrm{H} \mathrm{NMR}\left(400 \mathrm{MHz}, \mathrm{CDCl}_{3}\right) \delta 1.95(3 \mathrm{H}, \mathrm{dd}, J=6.7,1.6 \mathrm{~Hz})$, $2.32(1 \mathrm{H}, \mathrm{br}-\mathrm{t}, J=6.1 \mathrm{~Hz}), 3.85(1 \mathrm{H}, \mathrm{d}, J=4.1 \mathrm{~Hz}), 3.89(1 \mathrm{H}, \mathrm{d}, J=4.1 \mathrm{~Hz})$, 4.45 (1H, dd, $J=12.6,4.7 \mathrm{~Hz}), 4.57(1 \mathrm{H}, \mathrm{dd}, J=12.6,6.6 \mathrm{~Hz}), 6.34$ (1H, dq, J=15.8, $1.6 \mathrm{~Hz}), 6.59(1 \mathrm{H}$, $\mathrm{dq}, J=15.8,6.7 \mathrm{~Hz}) ;{ }^{13} \mathrm{C} \mathrm{NMR}\left(100 \mathrm{MHz} \mathrm{CDCl}_{3}\right) \delta 20.06,53.70,53.95,57.23,121.78,137.01$, 141.40, 142.34, 192.96, 193.78; FT-IR (neat) v 3437, 2935, 2856, 1695, 1684, 1628, 1362, 1286, 1007 , $858 \mathrm{~cm}^{-1}$.

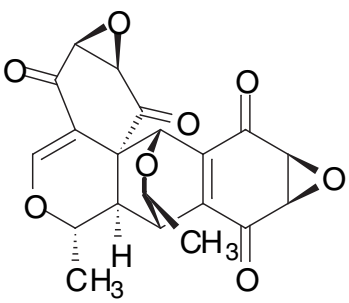

9

Epoxyquinone dimer 9. ${ }^{1}$ To a solution of $6(2.0 \mathrm{mg}, 0.010 \mathrm{mmol})$ in dry $\mathrm{CH}_{2} \mathrm{Cl}_{2}(0.4 \mathrm{ml})$ was added Dess-Martin periodinane $(5.7 \mathrm{mg}, 0.013 \mathrm{mmol})$ at $0{ }^{\circ} \mathrm{C}$ under argon atmosphere and stirred for $15 \mathrm{~min}$ at room temperature. The reaction mixture was quenched with saturated aqueous $\mathrm{NaHCO}_{3}$ and diluted with AcOEt. The organic phase was washed with saturated aqueous $\mathrm{NaHCO}_{3}$ and saturated aqueous $\mathrm{NaCl}$, and dried over anhydrous $\mathrm{Na}_{2} \mathrm{SO}_{4}$, then concentrated under reduced pressure. The residue was purified by silica gel column chromatography $($ AcOEt/Hexane $=1 / 10 \sim 1 / 3)$ to afford $9(1.4 \mathrm{mg}, 70 \%)$ as a colorless solid. 
${ }^{1} \mathrm{H}$ NMR $\left(400 \mathrm{MHz}, \mathrm{CDCl}_{3}\right) \delta 0.91(3 \mathrm{H}, \mathrm{d}, J=6.4 \mathrm{~Hz}), 1.09(3 \mathrm{H}, \mathrm{d}, J=6.8 \mathrm{~Hz}), 2.63(1 \mathrm{H}, \mathrm{d}, J=2.6 \mathrm{~Hz})$, $3.24(1 \mathrm{H}, \mathrm{dd}, J=2.6,1.6 \mathrm{~Hz}), 3.68(1 \mathrm{H}, \mathrm{d}, J=3.7 \mathrm{~Hz}), 3.83(1 \mathrm{H}, \mathrm{d}, J=3.7 \mathrm{~Hz}), 3.85(1 \mathrm{H}, \mathrm{d}, J=3.7 \mathrm{~Hz})$, $3.88(1 \mathrm{H}, \mathrm{d}, J=3.7 \mathrm{~Hz}), 4.37(1 \mathrm{H}, \mathrm{qd}, J=6.4,1.6 \mathrm{~Hz}), 4.61(1 \mathrm{H}, \mathrm{q}, J=6.8 \mathrm{~Hz}), 5.68(1 \mathrm{H}, \mathrm{s}), 7.87(1 \mathrm{H}, \mathrm{s})$; ${ }^{13} \mathrm{C}$ NMR $\left(100 \mathrm{MHz}, \mathrm{CDCl}_{3}\right) \delta 19.8,20.6,37.5,40.1,49.6,54.2,54.6,55.7,60.6,67.2,69.6,77.7$, 111.9, 143.5, 144.9, 159.3, 187.0, 187.6, 187.8, 199.8; FT-IR (neat) v 2968, 2925, 2852, 1716, 1695, $1684,1585,1308,1205,862,740 \mathrm{~cm}^{-1}$.<smiles></smiles>
$(9 \mathrm{H}, \mathrm{s}), 1.84(3 \mathrm{H}, \mathrm{d}, J=5.5 \mathrm{~Hz}), 3.46(3 \mathrm{H}, \mathrm{s}), 3.52(1 \mathrm{H}, \mathrm{dd}, J=3.8,1.1 \mathrm{~Hz}), 3.80(1 \mathrm{H}, \mathrm{dd}, J=3.8,1.6$ $\mathrm{Hz}), 4.35(1 \mathrm{H}, \mathrm{d}, J=13.8 \mathrm{~Hz}), 4.54(1 \mathrm{H}, \mathrm{d}, J=13.8 \mathrm{~Hz}), 4.80(1 \mathrm{H}, \mathrm{br}-\mathrm{s}), 5.98(1 \mathrm{H}$, br-d, $J=15.8 \mathrm{~Hz})$, $6.02(1 \mathrm{H}, \mathrm{dq}, J=15.8,5.5 \mathrm{~Hz}) ;{ }^{13} \mathrm{C}$ NMR $\left(100 \mathrm{MHz}, \mathrm{CDCl}_{3}\right) \delta-5.42,-5.37,18.30,19.14,25.87,53.46$, $54.03,56.85,60.49,70.65,121.74,131.52,134.89,146.27,194.62$; FT-IR (neat) v 2954, 2931, 2858, 1684, 1471, 1464, 1255, 1086, 837, $779 \mathrm{~cm}^{-1}$; HRMS (FAB) $[\mathrm{M}+\mathrm{H}]^{+}$calculated for $\mathrm{C}_{17} \mathrm{H}_{29} \mathrm{O}_{4} \mathrm{Si}$ : 325.1835, found: 325.1864 .

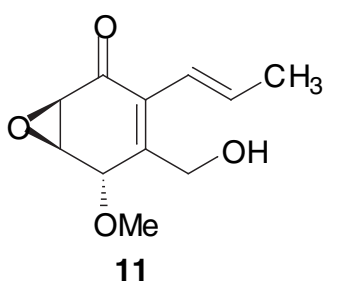

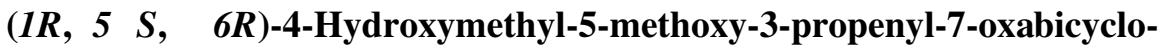
[4.1.0]hept-3-en-2-one (11). To a solution of $14(5.1 \mathrm{mg}, 0.016 \mathrm{mmol})$ in THF $(0.2 \mathrm{ml})$ was added TBAF (1.0 M THF solution, $18 \mathrm{ml}, 0.018 \mathrm{mmol})$ at $0{ }^{\circ} \mathrm{C}$ and stirred for $20 \mathrm{~min}$ at that temperature. The reaction mixture was concentrated under reduced pressure. The residue was purified by silica gel column chromatography (AcOEt/Hexane=1/10 1/1) to afford $\mathbf{1 1}$ $(2.3 \mathrm{mg}, 67 \%)$ as a colorless oil. ${ }^{1} \mathrm{H}$ NMR $\left(400 \mathrm{MHz}, \mathrm{CDCl}_{3}\right) \delta 1.85(3 \mathrm{H}$, d, $J=5.2 \mathrm{~Hz}), 2.00(1 \mathrm{H}, \mathrm{t}, J=5.4 \mathrm{~Hz}), 3.47(3 \mathrm{H}, \mathrm{s}), 3.56(1 \mathrm{H}, \mathrm{dd}, J=3.9,1.0$ $\mathrm{Hz}), 3.82(1 \mathrm{H}, \mathrm{dd}, J=3.9,1.5 \mathrm{~Hz}), 4.46(1 \mathrm{H}, \mathrm{dd}, J=14.0,5.4 \mathrm{~Hz}), 4.48(1 \mathrm{H}, \mathrm{dd}, J=14.0,5.4 \mathrm{~Hz}), 4.77$ $(1 \mathrm{H}, \mathrm{s}), 6.04(1 \mathrm{H}, \mathrm{br}-\mathrm{d}, J=16.0 \mathrm{~Hz}), 6.09(1 \mathrm{H}, \mathrm{dq}, J=16.0,5.2 \mathrm{~Hz}) ;{ }^{13} \mathrm{C}$ NMR $\left(100 \mathrm{MHz}, \mathrm{CDCl}_{3}\right) \delta$ 19.24, 53.68, 53.97, 56.21, 61.60, 72.41 121.40, 132.61, 135.53 144.54, 194.34; FT-IR (neat) v 3435, 2933, 2854, 1682, 1448, 1373, 1082, 1016, 968, $866 \mathrm{~cm}^{-1}$; HRMS (FAB) $[\mathrm{M}+\mathrm{H}]^{+}$calculated for $\mathrm{C}_{11} \mathrm{H}_{14} \mathrm{O}_{4}: 210.0892$, found: $210.0896 ;[\alpha]_{\mathrm{D}}^{20}=+197(c=0.17, \mathrm{MeOH})$.

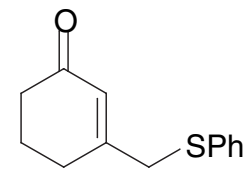

16

3-Phenylthiomethylcyclohex-2-en-1-one (16). To a solution of DABCO $(2.90 \mathrm{~g}, 25.8 \mathrm{mmol})$ and thioanisole $(3.00 \mathrm{ml}, 25.8 \mathrm{mmol})$ in dry THF $(39.0$ $\mathrm{ml})$ was added ${ }^{\mathrm{n}} \mathrm{BuLi}(1.56 \mathrm{M} n$-hexane solution, $19.6 \mathrm{ml}, 30.5 \mathrm{mmol})$ at $0{ }^{\circ} \mathrm{C}$ and stirred for 45 min under argon atmosphere. To the reaction mixture was added a solution of $\mathbf{1 5}(3.29 \mathrm{~g}, 23.5 \mathrm{mmol})$ in dry THF (10 ml) dropwise at 0 ${ }^{\circ} \mathrm{C}$ and warmed to room temperature. After $1.5 \mathrm{~h}$, the reaction mixture was cooled to $0{ }^{\circ} \mathrm{C}$ and quenched with $\mathrm{pH} 7$ phosphate buffer. The organic materials were extracted with AcOEt and the combined organic phase was washed with saturated aqueous $\mathrm{NaCl}$ and dried over anhydrous $\mathrm{Na}_{2} \mathrm{SO}_{4}$. After concentration under reduced pressure, the residue was purified by silica gel column chromatography (AcOEt/Hexane=1/50 1/3) to afford $\mathbf{1 6}$ $(3.65 \mathrm{~g}, 71 \%)$ as an yellow oil. ${ }^{1} \mathrm{H}$ NMR $\left(400 \mathrm{MHz}, \mathrm{CDCl}_{3}\right) \delta 1.89(2 \mathrm{H}$, quintet, $J=6.3 \mathrm{~Hz}), 2.22(2 \mathrm{H}$, t, $J=6.7 \mathrm{~Hz}), 2.36(2 \mathrm{H}, \mathrm{t}, J=5.9 \mathrm{~Hz}), 3.50(2 \mathrm{H}, \mathrm{s}), 5.67(1 \mathrm{H}, \mathrm{s}), 7.18-7.39(5 \mathrm{H}, \mathrm{m}) ;{ }^{13} \mathrm{C}$ NMR $(100$ $\left.\mathrm{MHz}, \mathrm{CDCl}_{3}\right) \delta 22.47,28.15,37.13,41.70,127.22,127.37,128.93,131.04,134.38,159.81,199.13$; FT-IR (neat) v 2947, 1666, 1623, 1581, 1481, 1439, 1371, 1346, 1325, 1255, 742, $692 \mathrm{~cm}^{-1}$; Anal. Calcd. for $\mathrm{C}_{13} \mathrm{H}_{14} \mathrm{OS}, \mathrm{C}: 71.52$, H: 6.46, O: 7.33, S: 14.69 Found C: 71.51, H: 6.48. 


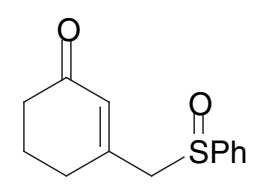

17

3-Phenylsulfinylmethylcyclohex-2-en-1-one (17). To a solution of $\mathbf{1 6}$ (3.65 $\mathrm{g}, 16.7 \mathrm{mmol})$ in dry $\mathrm{CH}_{2} \mathrm{Cl}_{2}(30 \mathrm{ml})$ was added mCPBA $(4.33 \mathrm{~g}, 25.1 \mathrm{mmol})$ at $0{ }^{\circ} \mathrm{C}$ and stirred for $1 \mathrm{~h}$ under argon atmosphere. The reaction mixture was quenched with saturated aqueous $\mathrm{NaHCO}_{3}$ and the organic materials were extracted with $\mathrm{CHCl}_{3}$. The combined organic phase was washed with saturated aqueous $\mathrm{NaCl}$ and dried over anhydrous $\mathrm{Na}_{2} \mathrm{SO}_{4}$. After concentration under reduced pressure, the residue was purified by silica gel column chromatography (AcOEt/Hexane $=1 / 5 \sim 1 / 0)$ to afford $17(3.65 \mathrm{~g}, 93 \%)$ as a colorless solid. ${ }^{1} \mathrm{H}$ NMR $\left(400 \mathrm{MHz}, \mathrm{CDCl}_{3}\right) \delta 1.91-2.01(2 \mathrm{H}, \mathrm{m}), 2.23-2.45(2 \mathrm{H}, \mathrm{m}), 3.59(1 \mathrm{H}, \mathrm{d}, J=12.3 \mathrm{~Hz}), 3.67(1 \mathrm{H}$, $\mathrm{d}, J=9.2 \mathrm{~Hz}), 5.69(1 \mathrm{H}, \mathrm{s}), 7.50-7.69(5 \mathrm{H}, \mathrm{m}) ;{ }^{13} \mathrm{C} \mathrm{NMR}\left(100 \mathrm{MHz}, \mathrm{CDCl}_{3}\right) \delta 22.19,30.50,36.83$, 64.97, 123.95, 129.20, 130.76, 131.57, 142.59, 152.65, 198.27; FT-IR (neat) v 2958, 1660, 1624, 1444, 1412, 1377, 1255, 1246, 1200, 1041, 748, $690 \mathrm{~cm}^{-1}$; Anal. calculated for $\mathrm{C}_{13} \mathrm{H}_{14} \mathrm{O}_{2} \mathrm{~S}, \mathrm{C}: 66.64, \mathrm{H}: 6.02$, O: 13.66, S: 13.68, found, C: 66.63, H: 6.03.

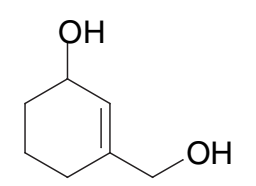

19

3-Hydroxymethylcyclohex-2-en-1-ol (19). To a solution of 17 (3.65 g, 15.6 $\mathrm{mmol})$ in dry $\mathrm{CH}_{2} \mathrm{Cl}_{2}(52 \mathrm{ml})$ was added 2,6-lutidine $(3.60 \mathrm{ml}, 31 \mathrm{mmol})$ and trifluoroacetic anhydride $(4.3 \mathrm{ml}, 31 \mathrm{mmol})$ at $0{ }^{\circ} \mathrm{C}$ under argon atmosphere and stirred for $1 \mathrm{~h}$. The reaction mixture was quenched with saturated aqueous $\mathrm{NaHCO}_{3}$ and stirred for 30 min at room temperature. The organic materials were extracted with $\mathrm{CHCl}_{3}$ and combined organic phase was washed with saturated aqueous $\mathrm{NaCl}$ and dried over anhydrous $\mathrm{Na}_{2} \mathrm{SO}_{4}$. After concentration under reduced pressure, the residue was filtered through a pad of silica gel $\left(\right.$ AcOEt/Hexane=1/10) and resulting aldehyde was obtained as crude product. ${ }^{1} \mathrm{H}$ NMR $(400 \mathrm{MHz}$, $\left.\mathrm{CDCl}_{3}\right) \delta 2.05(2 \mathrm{H}$, quintet, $J=6.4 \mathrm{~Hz}), 2.47(2 \mathrm{H}, \mathrm{td}, J=6.0,1.5 \mathrm{~Hz}), 2.52(2 \mathrm{H}, \mathrm{t}, J=6.7 \mathrm{~Hz}), 6.52(1 \mathrm{H}$, $\mathrm{s}), 9.75(1 \mathrm{H}, \mathrm{s})$.

To a solution of the aldehyde and $\mathrm{CeCl}_{3} \cdot 7 \mathrm{H}_{2} \mathrm{O}(18.0 \mathrm{~g}, 48.4 \mathrm{mmol})$ in $\mathrm{MeOH}(40 \mathrm{ml})$ was added $\mathrm{NaBH}_{4}(1.84 \mathrm{~g}, 48.4 \mathrm{mmol})$ at $-50{ }^{\circ} \mathrm{C}$ and stirred for $10 \mathrm{~min}$ at that temperature. The reaction mixture stirred for $10 \mathrm{~min}$ at room temperature and was quenched with saturated aqueous $\mathrm{NH}_{4} \mathrm{Cl}$. The organic materials were extracted with AcOEt $(4 \times 150 \mathrm{ml})$ and combined organic phase was dried over $\mathrm{Na}_{2} \mathrm{SO}_{4}$. After concentration under reduced pressure, the residue was purified by silica gel chromatography $(\mathrm{AcOEt} / \mathrm{Hexane}=1 / 5-1 / 0)$ to afford $19(1.60 \mathrm{~g}, 49 \%, 2$ steps $)$ as a colorless oil. ${ }^{1} \mathrm{H}$ NMR (400 MHz, CDCl $\left.{ }_{3}\right) \delta 1.52-1.63(2 \mathrm{H}, \mathrm{m}), 1.70-2.05(4 \mathrm{H}, \mathrm{m}), 2.50-3.05(2 \mathrm{H}, \mathrm{br}), 3.97(2 \mathrm{H}, \mathrm{s})$, $4.21(1 \mathrm{H}, \mathrm{br}-\mathrm{s}), 5.73(1 \mathrm{H}, \mathrm{s}) ;{ }^{13} \mathrm{C} \mathrm{NMR}\left(100 \mathrm{MHz}, \mathrm{CDCl}_{3}\right) \delta 18.89,25.30,31.58,65.17,65.41,123.78$, 140.63; FT-IR (neat) $v 3325,2933,2862,1344,1435,1162,1144 \mathrm{~cm}^{-1}$; Anal. calculated for $\mathrm{C}_{7} \mathrm{H}_{12} \mathrm{O}_{2}$, C: 65.60, H: 9.44, O: 24.97, found, C: 65.63, H: 9.43.

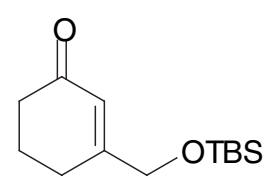

21

3-(tert-Butyldimethylsiloxymethyl)-cyclohex-2-en-1-one (21). To a solution of $19(140 \mathrm{mg}, 1.11 \mathrm{mmol}), \mathrm{Et}_{3} \mathrm{~N}(233 \mathrm{ml}, 1.67 \mathrm{mmol})$ and DMAP $(6.8 \mathrm{mg}$, $0.055 \mathrm{mmol})$ in dry $\mathrm{CH}_{2} \mathrm{Cl}_{2}(4.0 \mathrm{ml})$ was added TBSCl $(200 \mathrm{mg}, 1.33 \mathrm{mmol})$ at $0{ }^{\circ} \mathrm{C}$ and stirred for 40 min under argon atmosphere. The reaction mixture was quenched with saturated aqueous $\mathrm{NH}_{4} \mathrm{Cl}$ and diluted with AcOEt. The organic phase was washed with saturated aqueous $\mathrm{NaCl}$ and dried over anhydrous $\mathrm{Na}_{2} \mathrm{SO}_{4}$, then concentrated under reduced pressure. The residue was filtered through a pad of silica gel $(\mathrm{AcOEt} / \mathrm{Hexane}=1 / 5)$ to afford alcohol $\mathbf{2 0}$ and the crude product was used for next reaction without further purification. ${ }^{1} \mathrm{H}$ NMR $\left(400 \mathrm{MHz}, \mathrm{CDCl}_{3}\right) \delta 0.05$ $(6 \mathrm{H}, \mathrm{s}), 0.89(9 \mathrm{H}, \mathrm{s}), 1.47-1.69(2 \mathrm{H}, \mathrm{m}), 1.69-2.11(4 \mathrm{H}, \mathrm{m}), 4.00(2 \mathrm{H}, \mathrm{s}), 4.21(1 \mathrm{H}, \mathrm{s}), 5.74(1 \mathrm{H}, \mathrm{s})$.

To a solution of crude 20 in dry $\mathrm{CH}_{2} \mathrm{Cl}_{2}(3 \mathrm{ml})$ was added $\mathrm{MnO}_{2}(2.57 \mathrm{~g}, 75 \%, 22.2 \mathrm{mmol})$ at room temperature and stirred for $4 \mathrm{~h}$. The reaction mixture was filtered through a pad of Celite and the filtrate was concentrated and purified by silica gel chromatography (AcOEt/Hexane $=1 / 5-1 / 0)$ to afford 21 (252 mg, 68\%, 2 steps) as a colorless oil. ${ }^{1} \mathrm{H}$ NMR (400 MHz, $\left.\mathrm{CDCl}_{3}\right) \delta 0.09(6 \mathrm{H}, \mathrm{s}), 0.92(9 \mathrm{H}, \mathrm{s})$, $2.02(2 \mathrm{H}$, quintet, $J=6.3 \mathrm{~Hz}), 2.22(2 \mathrm{H}, \mathrm{t}, J=5.9 \mathrm{~Hz}), 2.41(2 \mathrm{H}, \mathrm{t}, J=6.3 \mathrm{~Hz}), 4.21-4.24(2 \mathrm{H}, \mathrm{m}), 6.13-$ $6.17(1 \mathrm{H}, \mathrm{m}) ;{ }^{13} \mathrm{C}$ NMR $\left(100 \mathrm{MHz}, \mathrm{CDCl}_{3}\right) \delta-5.58,18.20,22.46,25.73,25.87,37.85,64.98,123.07$, 164.10, 199.45; FT-IR (neat) v 2956, 2947, 2927, 2856, 1666, 1432, 1250, $839 \mathrm{~cm}^{-1}$; Anal. calculated for $\mathrm{C}_{13} \mathrm{H}_{24} \mathrm{O}_{2} \mathrm{Si}$, C: 64.95, H: 10.06, O: 13.31, Si: 11.68, found, C: 64.97, H: 10.06. 


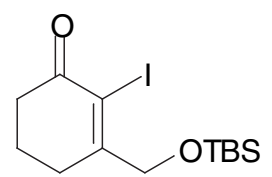

22

3-(tert-Butyldimethylsiloxymethyl)-2-iodocyclohex-2-en-1-one (22). To a solution of iodine $(2.19 \mathrm{~g}, 8.61 \mathrm{mmol})$ and pyridine $(1.53 \mathrm{ml}, 18.9 \mathrm{mmol})$ in dry $\mathrm{CH}_{2} \mathrm{Cl}_{2}(6.0 \mathrm{ml})$ was added $\mathrm{PhI}\left(\mathrm{OCOCF}_{3}\right)_{2}(3.70 \mathrm{~g}, 8.61 \mathrm{mmol})$ at room temperature and stirred for $15 \mathrm{~min}$ under argon atmosphere. To the reaction mixture was added $21(2.07 \mathrm{~g}, 8.61 \mathrm{mmol})$ and stirred for $36 \mathrm{~h}$ in the dark. The reaction mixture was quenched with saturated aqueous $\mathrm{NaHCO}_{3}$ and diluted with AcOEt. The organic phase was washed with saturated aqueous $\mathrm{Na}_{2} \mathrm{~S}_{2} \mathrm{O}_{3}$ and saturated aqueous $\mathrm{NaCl}$, dried over anhydrous $\mathrm{Na}_{2} \mathrm{SO}_{4}$. After concentration under reduced pressure, the residue was purified by silica gel column chromatography (AcOEt/Hexane=0/1 1/10) to afford $22(2.59 \mathrm{~g}, 82 \%)$ as a colorless oil. ${ }^{1} \mathrm{H}$ NMR $\left(400 \mathrm{MHz}, \mathrm{CDCl}_{3}\right)$ $\delta 0.12(6 \mathrm{H}, \mathrm{s}), 0.93(9 \mathrm{H}, \mathrm{s}), 2.01(2 \mathrm{H}$, quintet, $J=6.3 \mathrm{~Hz}), 2.67-2.71(4 \mathrm{H}, \mathrm{m}), 4.44(2 \mathrm{H}, \mathrm{s}) ;{ }^{13} \mathrm{C} \mathrm{NMR}$ $\left(100 \mathrm{MHz}, \mathrm{CDCl}_{3}\right) \delta-5.53,18.05,22.03,25.67,29.53,37.04,72.00,102.40,168.43,191.33$; FT-IR (neat) $v 2952,2929,2856,1685,1589,1471,1462,1257,1130,1107,837,779 \mathrm{~cm}^{-1}$; HRMS (FAB) $[\mathrm{M}+\mathrm{H}]^{+}$calculated for $\mathrm{C}_{13} \mathrm{H}_{24} \mathrm{IO}_{2} \mathrm{Si}$ : 376.0590, found: 376.0609 .<smiles>C/C=C/C1=C(CO)CCCC1=O</smiles>

10

3-Hydroxymethyl-2-(1-propenyl)cyclohex-2-en-1-one (10). Under argon atmosphere, to a solution of $22(166 \mathrm{mg}, 0.453 \mathrm{mmol}),(E)-1$ propenylborate $(190 \mathrm{mg}, 2.21 \mathrm{mmol}), \mathrm{Ag}_{2} \mathrm{O}(520 \mathrm{mg}, 2.27 \mathrm{mmol})$ and $\mathrm{Ph}_{3} \mathrm{As}(13.9 \mathrm{mg}, 0.046 \mathrm{mmol})$ in THF- $\mathrm{H}_{2} \mathrm{O}(8: 1,7.0 \mathrm{ml})$ was added $\mathrm{Pd}(\mathrm{PhCN})_{2} \mathrm{Cl}_{2}(8.7 \mathrm{mg}, 0.023 \mathrm{mmol})$ at room temperature in the dark and stirred for $14 \mathrm{~h}$. The reaction mixture was quenched with saturated aqueous $\mathrm{NH}_{4} \mathrm{Cl}$ and stirred for $30 \mathrm{~min}$ at that temperature. The reaction mixture was filtered through a pad of Celite and the organic materials were extracted with $\mathrm{CHCl}_{3}(3 \mathrm{x}$ $30 \mathrm{ml}$ ). The combined organic phase was washed with saturated aqueous $\mathrm{NaCl}$ and dried over anhydrous $\mathrm{Na}_{2} \mathrm{SO}_{4}$. After concentration under reduced pressure, the residue was filtered through a pad of silica gel $(\mathrm{AcOEt} / \mathrm{Hexane}=1 / 10)$ and resulting dienone $(130 \mathrm{mg})$ was obtained as crude product. ${ }^{1} \mathrm{H}$ NMR (400 MHz, CDCl $)_{3} \delta 0.05(6 \mathrm{H}, \mathrm{s}), 0.89(9 \mathrm{H}, \mathrm{s}), 1.78-1.86(2 \mathrm{H}, \mathrm{m}), 2.40(2 \mathrm{H}, \mathrm{t}, J=6.1 \mathrm{~Hz}), 2.50$ $(2 \mathrm{H}, \mathrm{t}, J=5.7 \mathrm{~Hz}), 4.41(2 \mathrm{H}, \mathrm{s}), 5.68(1 \mathrm{H}, \mathrm{dq}, J=15.8,6.6 \mathrm{~Hz}), 6.01-6.11(1 \mathrm{H}, \mathrm{m})$.

To a solution of the silyl ether $(130 \mathrm{mg})$ in THF $(1.0 \mathrm{ml})$ was added TBAF (1.0 M THF solution, $0.50 \mathrm{ml}, 0.50 \mathrm{mmol}$ ) at $0{ }^{\circ} \mathrm{C}$ and stirred for $20 \mathrm{~min}$ at that temperature. The reaction mixture was concentrated under reduced pressure. The residue was purified by silica gel column chromatography (AcOEt/Hexane=1/5 1/1) to afford 10 (50.5 mg, 67\%, 2 steps) as a colorless oil. ${ }^{1} \mathrm{H}$ NMR (400 MHz, $\left.\mathrm{CDCl}_{3}\right) \delta 1.80(1 \mathrm{H}, \mathrm{br}-\mathrm{s}), 1.83(3 \mathrm{H}, \mathrm{dd}, J=6.5,1.5 \mathrm{~Hz}), 1.97(2 \mathrm{H}$, quintet $J=6.4 \mathrm{~Hz}), 2.43(2 \mathrm{H}, \mathrm{t}, J=6.6$ $\mathrm{Hz}), 2.55(2 \mathrm{H}, \mathrm{t}, J=6.0 \mathrm{~Hz}), 4.44(2 \mathrm{H}, \mathrm{d}, J=4.6 \mathrm{~Hz}), 5.74(1 \mathrm{H}, \mathrm{dq}, J=15.9,6.5 \mathrm{~Hz}), 6.10(1 \mathrm{H}, \mathrm{dq}$, $J=15.9,1.1 \mathrm{~Hz}) ;{ }^{13} \mathrm{C}$ NMR $\left(100 \mathrm{MHz}, \mathrm{CDCl}_{3}\right) \delta 16.7,18.9,21.8,27.6,38.2,63.24,123.2,132.4$, 133.5, 156.4, 199.5; FT-IR (neat) v 3419, 2935, 2870, 1668, 1651, 1450, 1429, 1363, 1053, 974, 565 $\mathrm{cm}^{-1}$; HRMS (FAB) $[\mathrm{M}+\mathrm{H}]^{+}$calculated for $\mathrm{C}_{10} \mathrm{H}_{15} \mathrm{O}_{2}: 167.1072$, found: 167.1065 .

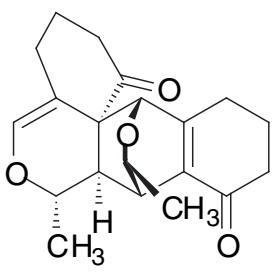

25

Dimer 25. To a solution of $\mathbf{1 0}(2.0 \mathrm{mg}, 0.012 \mathrm{mmol})$ in dry $\mathrm{CH}_{2} \mathrm{Cl}_{2}(0.3$ ml) was added $\mathrm{MnO}_{2}(88 \mathrm{mg}, 75 \%, 0.76 \mathrm{mmol})$ at $0{ }^{\circ} \mathrm{C}$ under argon atmosphere and stirred for $1.5 \mathrm{~h}$ at that temperature. The reaction mixture was filtered through a pad of Celite and concentrated in vacuo. The residue was allowed to stand at room temperature for $10 \mathrm{~h}$ and purified by preparative thin-layer chromatography (AcOEt/Hexane=1/1) to afford $\mathbf{2 5}$ $(1.4 \mathrm{mg}, 70 \%)$ as a colorless oil. ${ }^{1} \mathrm{H}$ NMR $\left(400 \mathrm{MHz}, \mathrm{CDCl}_{3}\right) \delta 0.83(3 \mathrm{H}$, d, $J=6.2 \mathrm{~Hz}), 1.08(3 \mathrm{H}, \mathrm{d}, J=6.6 \mathrm{~Hz}), 1.93-2.09(4 \mathrm{H}, \mathrm{m}), 2.14-2.62(9 \mathrm{H}$, m), $3.17(1 \mathrm{H}, \mathrm{s}), 4.34(1 \mathrm{H}, \mathrm{q}, J=6.6 \mathrm{~Hz}), 4.44(1 \mathrm{H}, \mathrm{q}, J=6.2 \mathrm{~Hz}), 4.60(1 \mathrm{H}, \mathrm{s}), 6.38(1 \mathrm{H}, \mathrm{br}-\mathrm{s}) ;{ }^{13} \mathrm{C}$ NMR $\left(100 \mathrm{MHz}, \mathrm{CDCl}_{3}\right) \delta 16.7,18.9,21.8,27.6,38.2,63.24,123.2,132.4,133.5,156.4,199.5$; FTIR (neat) $v$ 3419, 2935, 2870, 1668, 1651, 1450, 1429, 1363, 1053, 974, $565 \mathrm{~cm}^{-1}$; HRMS (FAB) $[\mathrm{M}+\mathrm{H}]^{+}$calculated for $\mathrm{C}_{10} \mathrm{H}_{15} \mathrm{O}_{2}: 167.1072$, found: 167.1065 .

General procedure of the oxidative dimerization of diol 3 in various solvents (Table 5). To a solution of $3(5.0 \mathrm{mg}, 0.025 \mathrm{mmol})$ in dry $\mathrm{CH}_{2} \mathrm{Cl}_{2}(0.3 \mathrm{ml})$ was added $\mathrm{MnO}_{2}(30 \mathrm{mg}, 75 \%, 0.25 \mathrm{mmol})$ at $0{ }^{\circ} \mathrm{C}$ under argon atmosphere and stirred for $20 \mathrm{~min}$ at that temperature. The reaction mixture was filtered through a pad of Celite and washed with AcOEt. The filtrate was concentrated at $0{ }^{\circ} \mathrm{C}$ in vacuo and a solvent $(0.5 \mathrm{ml})$ was added. The reaction mixture was allowed to stand at room 
temperature for indicated period and concentrated in vacuo. The residue was purified by preparative thin-layer chromatography (AcOEt/Hexane=1/1) to afford epoxyquinol A(1) and epoxyquinol B (2).

References

(1) Li, C.; Bardhan, S.; Pace, E. A.; Liang, M.-C.; Gilmore. T. D.; Porco, J. A. Jr. Org. Lett. 2002, 4, 3267. 


\section{Theoretical calculations}

All calculations were performed with TITAN 1.0.5, Schrödinger Inc. and Wavefunction Inc. All structures were optimized and subjected to frequency analysis with the B3LYP/6-31G* method, followed by single point B3LYP/6-31G* calculation.

\begin{tabular}{|c|c|c|c|}
\hline $\begin{array}{l}\text { TS } 26 \text { to } 2 \\
\text { E(B3LYP/ } \\
v=-424.05 \\
\text { Cartesian }\end{array}$ & $\begin{array}{l}\left.6-31 \mathrm{G}^{*}\right)=-727.16 \\
\mathrm{~cm}^{-1} \\
\text { Coordinates (Angs }\end{array}$ & $\begin{array}{l}44 \text { au } \\
\text { oms) }\end{array}$ & \\
\hline Atom & $\mathrm{Y}$ & & \\
\hline $1 \mathrm{H} \mathrm{H} 1$ & -1.157139228 & -1.946974900 & 1.950378038 \\
\hline $2 \mathrm{C} \mathrm{C} 2$ & -0.811937015 & -1.113701575 & 2.555763828 \\
\hline $3 \mathrm{H} \mathrm{H} 3$ & 0.456777966 & -0.627567673 & 4.181442986 \\
\hline $4 \mathrm{C} \mathrm{C} 4$ & 0.407701139 & -1.172612416 & 3.241403650 \\
\hline $5 \mathrm{C} \mathrm{C} 5$ & -1.502816853 & 0.097110277 & 2.530885418 \\
\hline 6 C C6 & -0.839638690 & 1.326518678 & 2.780514719 \\
\hline $7 \mathrm{C} \mathrm{C7}$ & 0.558310522 & 1.381669875 & 2.527752479 \\
\hline $8 \mathrm{H} \mathrm{H} 8$ & 0.926178709 & 2.341381631 & 2.132600826 \\
\hline 9009 & 1.401081358 & 0.447440321 & 2.570672596 \\
\hline $10 \mathrm{C} \mathrm{C} 10$ & 1.376194428 & -2.304359138 & 3.105454692 \\
\hline 11 H H11 & 2.400579286 & -1.938190562 & 3.224087338 \\
\hline 12 H H12 & 1.286625823 & -2.805780064 & 2.137853225 \\
\hline 13 H H13 & 1.197781820 & -3.041861375 & 3.900247483 \\
\hline $14 \mathrm{C} \mathrm{C} 14$ & -2.912714723 & 0.098588427 & 2.015926134 \\
\hline $15 \mathrm{C} \mathrm{C} 15$ & -1.608592350 & 2.623513725 & 2.879324342 \\
\hline 16 H H16 & -0.944089392 & 3.456895478 & 2.590170440 \\
\hline $17 \mathrm{C} \mathrm{C} 17$ & -3.491451583 & 1.409021764 & 1.571780999 \\
\hline 18 H H18 & -4.553012766 & 1.381373272 & 1.334330601 \\
\hline 19 C C19 & -2.838502306 & 2.664103823 & 1.988862752 \\
\hline 20 H H20 & -3.456252423 & 3.555849937 & 2.094584009 \\
\hline $21 \mathrm{O} \mathrm{O} 21$ & -3.580664793 & -0.921068711 & 1.967369064 \\
\hline $22 \mathrm{O} \mathrm{O} 22$ & -2.672644052 & 2.180128752 & 0.664437975 \\
\hline $23 \mathrm{O} \mathrm{O} 23$ & -2.121326710 & 2.869229868 & 4.191804907 \\
\hline $24 \mathrm{C} \mathrm{C} 24$ & -1.128164564 & 3.168711981 & 5.153866262 \\
\hline $25 \mathrm{H} \mathrm{H} 25$ & -0.541798030 & 4.055945327 & 4.865147396 \\
\hline $26 \mathrm{H} \mathrm{H} 26$ & -0.436575629 & 2.328702127 & 5.308345411 \\
\hline 27 H H27 & -1.651766314 & 3.377061667 & 6.090162940 \\
\hline
\end{tabular}




\begin{tabular}{|c|c|c|c|}
\hline $\begin{array}{l}\text { TS } 26 \text { to } 27 \\
v=-451.85 \\
\text { Cartesian }\end{array}$ & $\begin{array}{l}\left.-31 \mathrm{G}^{*}\right)=-727.156 \\
\mathrm{~cm}^{-1} \\
\text { coordinates (Angs }\end{array}$ & $\begin{array}{l}69 \text { au } \\
\text { oms) }\end{array}$ & \\
\hline Atom & $\begin{array}{lll}X & Y & Z\end{array}$ & & \\
\hline $1 \mathrm{H} \mathrm{H} 1$ & -1.446516336 & -1.921256686 & 3.004380774 \\
\hline $2 \mathrm{C} \mathrm{C} 2$ & -0.837401806 & -1.164438272 & 2.517897033 \\
\hline $3 \mathrm{C} \mathrm{C} 3$ & 0.518125985 & -1.381513768 & 2.237426080 \\
\hline $4 \mathrm{H} \mathrm{H} 4$ & 0.901252144 & -0.925137236 & 1.327575074 \\
\hline $5 \mathrm{C} \mathrm{C} 5$ & -1.372205121 & 0.107021815 & 2.308807982 \\
\hline 6 C C6 & -0.553090826 & 1.263047866 & 2.277869596 \\
\hline $7 \mathrm{C} \mathrm{C7}$ & 0.723164927 & 1.185022622 & 2.901714668 \\
\hline $8 \mathrm{H} \mathrm{H} 8$ & 1.069362046 & 2.111907465 & 3.379918640 \\
\hline 9 O O9 & 1.436432233 & 0.163039222 & 3.096716921 \\
\hline $10 \mathrm{C} \mathrm{C} 10$ & 1.265272685 & -2.590818418 & 2.707415690 \\
\hline 11 H H11 & 2.318212516 & -2.341855721 & 2.870708547 \\
\hline 12 H H12 & 1.228205582 & -3.372965784 & 1.936762261 \\
\hline 13 H H13 & 0.846767180 & -2.994200414 & 3.633803265 \\
\hline 14 C C14 & -2.865321419 & 0.256591895 & 2.343113892 \\
\hline $15 \mathrm{O} 015$ & -3.602790318 & -0.569722118 & 2.854396666 \\
\hline 16 C C16 & -3.424456928 & 1.490117611 & 1.699805690 \\
\hline 17 H H17 & -4.506414127 & 1.594380402 & 1.756053766 \\
\hline $18 \mathrm{C} \mathrm{C} 18$ & -1.1195886 & 3 & 2.090266666 \\
\hline 19 H H19 & -0.50543 & 29990 & 1.357004142 \\
\hline $20 \mathrm{C} C 20$ & -2.547955716 & 2.675194950 & 1.568438982 \\
\hline $21 \mathrm{H} \mathrm{H} 21$ & -3.012358223 & 3.662406817 & 1.551055570 \\
\hline $22 \mathrm{O} O 22$ & -2.823043140 & 1.855207565 & 0.441201853 \\
\hline $23 \mathrm{O} \mathrm{O} 23$ & -1.018268948 & 3.501665758 & 3.252656387 \\
\hline 24 C C24 & -1.709929005 & 3.050293864 & 4.410537997 \\
\hline $25 \mathrm{H} \mathrm{H} 25$ & -1.368306039 & 2.058629607 & 4.735654186 \\
\hline 26 H H26 & -2.797525008 & 3.015298701 & 4.259332549 \\
\hline 27 H H27 & -1.487975185 & 3.776596969 & 5.195966684 \\
\hline
\end{tabular}




\begin{tabular}{|c|c|c|c|}
\hline $\begin{array}{l}\text { E(B3LYP/ } \\
v=-381.12 \\
\text { Cartesian }\end{array}$ & $\begin{array}{l}\left.5-31 \mathrm{G}^{*}\right)=-686.65 \\
\mathrm{~cm}^{-1} \\
\text { Coordinates (Angs }\end{array}$ & $\begin{array}{l}85 \mathrm{au} \\
\text { oms) }\end{array}$ & \\
\hline Atom & $\mathrm{Y}$ & & \\
\hline $1 \mathrm{H} \mathrm{H} 1$ & -0.908043601 & -1.958005172 & 1.861426824 \\
\hline $2 \mathrm{C} \mathrm{C} 2$ & -0.708951233 & -1.131908968 & 2.538374225 \\
\hline $3 \mathrm{H} \mathrm{H} 3$ & 0.137607452 & -0.714014187 & 4.439962355 \\
\hline $4 \mathrm{C} \mathrm{C} 4$ & 0.314750352 & -1.213758415 & 3.491244622 \\
\hline $5 \mathrm{C} \mathrm{C} 5$ & -1.409343769 & 0.062736950 & 2.385734813 \\
\hline 6 C C6 & -0.864629973 & 1.294215915 & 2.833057498 \\
\hline $7 \mathrm{C} \mathrm{C7}$ & 0.556747386 & 1.417128915 & 2.987538287 \\
\hline 8 H H8 & 0.957473390 & 2.422335552 & 2.802450747 \\
\hline 9009 & 1.388395503 & 0.502220459 & 3.179798028 \\
\hline $10 \mathrm{C} \mathrm{C} 10$ & 1.319586248 & -2.318604371 & 3.526577665 \\
\hline 11 H H11 & 2.281429740 & -1.937848873 & 3.884093773 \\
\hline 12 H H12 & 1.458566437 & -2.781093480 & 2.545779793 \\
\hline $13 \mathrm{H} \mathrm{H} 13$ & 0.992646145 & -3.090752091 & 4.237094626 \\
\hline 14 C C14 & -2.720334436 & 0.024453851 & 1.648866900 \\
\hline $15 \mathrm{C} \mathrm{C} 15$ & -1.610756737 & 2.561022789 & 2.760181834 \\
\hline $16 \mathrm{C} \mathrm{C} 16$ & -3.404129599 & 1.330054692 & 1.349319063 \\
\hline 17 H H17 & -4.455925032 & 1.242481931 & 1.084537731 \\
\hline 18 C C18 & -2.873297069 & 2.581139506 & 1.932645528 \\
\hline 19 H H19 & -3.531969396 & 3.431298023 & 2.101110455 \\
\hline $20 \mathrm{O} O 20$ & -3.249868207 & -1.029480939 & 1.341791996 \\
\hline 210021 & -2.639784383 & 2.245535505 & 0.554496320 \\
\hline $22 \mathrm{O} O 22$ & -1.223883106 & 3.596024420 & 3.288908610 \\
\hline
\end{tabular}




\begin{tabular}{|c|c|c|c|}
\hline $\begin{array}{l}\text { TS } 7 \text { to } \mathbf{8 b} \\
\text { E(B3LYP/C } \\
v=-347.02 \\
\text { Cartesian }\end{array}$ & $\begin{array}{l}6-31 \mathrm{G}^{*} \text { )=-686.664 } \\
\mathrm{cm}^{-1} \\
\text { Coordinates (Angs }\end{array}$ & $\begin{array}{l}1 \mathrm{au} \\
\mathrm{oms})\end{array}$ & \\
\hline Atom & $\begin{array}{lll}X & Y & Z\end{array}$ & & \\
\hline $1 \mathrm{H} \mathrm{H} 1$ & -1.474911498 & -1.963588624 & 2.942395520 \\
\hline $2 \mathrm{C} \mathrm{C} 2$ & -0.858149460 & -1.165165679 & 2.538280085 \\
\hline $3 \mathrm{C} \mathrm{C} 3$ & 0.508336105 & -1.352877143 & 2.297086341 \\
\hline $4 \mathrm{H} \mathrm{H} 4$ & 0.938204891 & -0.801931019 & 1.465317042 \\
\hline $5 \mathrm{C} \mathrm{C} 5$ & -1.405429976 & 0.105980289 & 2.394782364 \\
\hline $6 \mathrm{C} \mathrm{C6}$ & -0.601477395 & 1.275295509 & 2.465082258 \\
\hline $7 \mathrm{C} \mathrm{C} 7$ & 0.654323603 & 1.203967688 & 3.158585280 \\
\hline $8 \mathrm{H} \mathrm{H} 8$ & 0.953667179 & 2.131910144 & 3.663025107 \\
\hline 9009 & 1.370110057 & 0.194313424 & 3.335839096 \\
\hline $10 \mathrm{C} \mathrm{C} 10$ & 1.257173736 & -2.575894462 & 2.715420498 \\
\hline 11 H H11 & 2.287014462 & -2.313013140 & 2.976394613 \\
\hline 12 H H12 & 1.305585971 & -3.283079249 & 1.875480548 \\
\hline 13 H H13 & 0.787111787 & -3.075258477 & 3.566894919 \\
\hline 14 C C14 & -2.904839617 & 0.247455918 & 2.424282659 \\
\hline $15 \mathrm{O} 015$ & -3.636369298 & -0.611107623 & 2.883268298 \\
\hline $16 \mathrm{C} \mathrm{C} 16$ & -3.461954146 & 1.507792652 & 1.825511739 \\
\hline 17 H H17 & -4.542599092 & 1.621080769 & 1.884714739 \\
\hline 18 C C18 & -1.188540379 & 2.616665592 & 2.329480546 \\
\hline 19 C C19 & -2.577150623 & 2.697991471 & 1.739579903 \\
\hline 20 H H20 & -3.011798618 & 3.696125922 & 1.734054661 \\
\hline 210021 & -2.845799974 & 1.892811835 & 0.590420665 \\
\hline 22 O O22 & -0.630960280 & 3.651361358 & 2.680361940 \\
\hline
\end{tabular}




\begin{tabular}{|c|c|c|c|}
\hline $\begin{array}{l}\text { TS } 23 \text { to } 2 \\
\text { E(B3LYP/ } \\
v=-468.58 \\
\text { Cartesian }\end{array}$ & $\begin{array}{l}\left.5-31 \mathrm{G}^{*}\right)=-538.676 \\
\mathrm{~cm}^{-1} \\
\text { Coordinates (Angs }\end{array}$ & & \\
\hline Atom & $\mathrm{X}$ & & \\
\hline $1 \mathrm{H} \mathrm{H} 1$ & -0.947193068 & -1.983150287 & 2.068644705 \\
\hline $2 \mathrm{C} \mathrm{C} 2$ & -0.798089458 & -1.146176215 & 2.744495811 \\
\hline 3 H H3 & -0.082693855 & -0.649982500 & 4.676519027 \\
\hline $4 \mathrm{C} \mathrm{C} 4$ & 0.163309314 & -1.187210504 & 3.762744227 \\
\hline 5 C C5 & -1.456560433 & 0.060311149 & 2.502686105 \\
\hline $6 \mathrm{C} \mathrm{C6}$ & -0.896957767 & 1.300820361 & 2.910979340 \\
\hline $7 \mathrm{C} \mathrm{C7}$ & 0.510772224 & 1.343544895 & 3.089320562 \\
\hline 8 H H8 & 0.996729116 & 2.283240002 & 2.785627204 \\
\hline 9009 & 1.292987960 & 0.404772038 & 3.411070708 \\
\hline $10 \mathrm{C} \mathrm{C} 10$ & 1.131873566 & -2.316749341 & 3.929509648 \\
\hline 11 H H11 & 2.067355736 & -1.949687091 & 4.362478036 \\
\hline 12 H H12 & 1.349501153 & -2.810256636 & 2.978150687 \\
\hline 13 H H13 & 0.718325921 & -3.061829234 & 4.622901860 \\
\hline 14 C C14 & -2.649895103 & 0.046767975 & 1.581930614 \\
\hline $15 \mathrm{C} \mathrm{C} 15$ & -1.641002960 & 2.598863707 & 2.678979676 \\
\hline $16 \mathrm{C} \mathrm{C} 16$ & -3.381052931 & 1.361180407 & 1.349231272 \\
\hline 17 H H17 & -4.123027190 & 1.460670128 & 2.158161768 \\
\hline $18 \mathrm{C} \mathrm{C} 18$ & -2.446686478 & 2.578464516 & 1.371157563 \\
\hline 19 H H19 & -3.029259925 & 3.501266811 & 1.259674256 \\
\hline $20 \mathrm{O} O 20$ & -3.029075030 & -0.991388397 & 1.064395614 \\
\hline $21 \mathrm{H} \mathrm{H} 21$ & -3.941787507 & 1.270586362 & 0.413584476 \\
\hline $22 \mathrm{H} \mathrm{H} 22$ & -1.755559355 & 2.527865670 & 0.518763696 \\
\hline $23 \mathrm{H} \mathrm{H} 23$ & -2.332848176 & 2.784985048 & 3.515893369 \\
\hline 24 H H 24 & -0.933825158 & 3.438205952 & 2.679712639 \\
\hline
\end{tabular}




\begin{tabular}{|c|c|c|c|}
\hline $\begin{array}{l}\text { E(B3LYP/ } \\
v=-438.37 \\
\text { Cartesian }\end{array}$ & $\begin{array}{l}\left.5-31 \mathrm{G}^{*}\right)=-687.852 \\
\mathrm{~cm}^{-1} \\
\text { Coordinates (Angs }\end{array}$ & $\begin{array}{l}05 \mathrm{au} \\
\text { oms) }\end{array}$ & \\
\hline Atom & $\mathrm{X}$ & & \\
\hline $1 \mathrm{H} \mathrm{H} 1$ & -1.058891961 & -1.908128546 & 2.006715385 \\
\hline $2 \mathrm{C} \mathrm{C} 2$ & -0.723513496 & -1.046702422 & 2.577049232 \\
\hline $3 \mathrm{H} \mathrm{H} 3$ & 0.535954540 & -0.490573485 & 4.189186275 \\
\hline $4 \mathrm{C} \mathrm{C} 4$ & 0.497573121 & -1.063431506 & 3.265007855 \\
\hline 5 C C5 & -1.429500544 & 0.154251285 & 2.499862258 \\
\hline 6 C C6 & -0.778842444 & 1.401610376 & 2.693815630 \\
\hline $7 \mathrm{C} \mathrm{C7}$ & 0.621941781 & 1.456904610 & 2.449143341 \\
\hline $8 \mathrm{H} \mathrm{H} 8$ & 0.988529836 & 2.397130216 & 2.011397067 \\
\hline 9009 & 1.467725962 & 0.529671463 & 2.541394768 \\
\hline $10 \mathrm{C} \mathrm{C} 10$ & 1.477585323 & -2.190260057 & 3.171174824 \\
\hline 11 H H11 & 2.497290987 & -1.814082627 & 3.297790211 \\
\hline $12 \mathrm{H} \mathrm{H} 12$ & 1.406977130 & -2.715399648 & 2.214678348 \\
\hline $13 \mathrm{H} \mathrm{H} 13$ & 1.291215587 & -2.910044602 & 3.980267354 \\
\hline $14 \mathrm{C} \mathrm{C} 14$ & -2.850120069 & 0.110480939 & 2.016224961 \\
\hline $15 \mathrm{C} \mathrm{C} 15$ & -1.543896387 & 2.701624801 & 2.745470872 \\
\hline $16 \mathrm{H} \mathrm{H} 16$ & -0.916748457 & 3.508301902 & 2.347576018 \\
\hline $17 \mathrm{C} \mathrm{C} 17$ & -3.482166379 & 1.399474882 & 1.583095914 \\
\hline 18 H H18 & -4.555171067 & 1.342347950 & 1.409321914 \\
\hline 19 C C19 & -2.832877789 & 2.679098794 & 1.938990731 \\
\hline 20 H H 20 & -3.464993580 & 3.559226827 & 2.060654397 \\
\hline $21 \mathrm{O} O 21$ & -3.492425586 & -0.927070690 & 2.004859826 \\
\hline 220022 & -2.739739039 & 2.168676462 & 0.614694120 \\
\hline $23 \mathrm{O} \mathrm{O} 23$ & -1.841655765 & 3.113992963 & 4.088292964 \\
\hline $24 \mathrm{H} \mathrm{H} 24$ & -2.172218138 & 2.332431608 & 4.559528999 \\
\hline
\end{tabular}




\begin{tabular}{|c|c|c|c|}
\hline $\begin{array}{l}\text { IS } 4 \text { to 5b } \\
\text { v=-445.02 } \\
\text { Cartesian }\end{array}$ & $\begin{array}{l}\left.5-31 \mathrm{G}^{*}\right)=-687.851 \\
\mathrm{~cm}^{-1} \\
\text { Coordinates (Angs }\end{array}$ & $\begin{array}{l}95 \mathrm{au} \\
\text { oms) }\end{array}$ & \\
\hline Atom & $\mathrm{Y}$ & & \\
\hline $1 \mathrm{H} \mathrm{H} 1$ & -1.458905111 & -1.885049549 & 3.061806744 \\
\hline $2 \mathrm{C} \mathrm{C} 2$ & -0.851542103 & -1.138797371 & 2.557405864 \\
\hline $3 \mathrm{C} \mathrm{C} 3$ & 0.499835723 & -1.365150557 & 2.263495085 \\
\hline $4 \mathrm{H} \mathrm{H} 4$ & 0.874485630 & -0.912601350 & 1.347990141 \\
\hline 5 C C5 & -1.378166459 & 0.132466497 & 2.330567264 \\
\hline $6 \mathrm{C} \mathrm{C6}$ & -0.545893225 & 1.279263701 & 2.267677541 \\
\hline $7 \mathrm{C} \mathrm{C7}$ & 0.728815003 & 1.202177588 & 2.897883003 \\
\hline $8 \mathrm{H} \mathrm{H} 8$ & 1.080295160 & 2.133328126 & 3.363228927 \\
\hline 9009 & 1.431736952 & 0.177374415 & 3.111630823 \\
\hline $10 \mathrm{C} \mathrm{C} 10$ & 1.246995387 & -2.576751147 & 2.726695619 \\
\hline 11 H H11 & 2.303252682 & -2.333353646 & 2.875848814 \\
\hline 12 H H12 & 1.196074219 & -3.358583017 & 1.956473416 \\
\hline $13 \mathrm{H} \mathrm{H} 13$ & 0.838144717 & -2.978172807 & 3.658113339 \\
\hline $14 \mathrm{C} \mathrm{C} 14$ & -2.867848504 & 0.304378059 & 2.393916048 \\
\hline 150015 & -3.603190693 & -0.495540922 & 2.948474441 \\
\hline $16 \mathrm{C} \mathrm{C} 16$ & -3.422545647 & 1.528195441 & 1.729973859 \\
\hline 17 H H17 & -4.500461310 & 1.654305835 & 1.810504227 \\
\hline $18 \mathrm{C} \mathrm{C} 18$ & -1.100950155 & 2.676770680 & 2.046967022 \\
\hline 19 H H19 & -0.475621324 & 3.211320678 & 1.320886012 \\
\hline $20 \mathrm{C} \mathrm{C} 20$ & -2.529015836 & 2.691261557 & 1.527912755 \\
\hline $21 \mathrm{H} \mathrm{H} 21$ & -2.977700622 & 3.684698186 & 1.489349986 \\
\hline $22 \mathrm{O} O 22$ & -2.848822633 & 1.835198748 & 0.441357696 \\
\hline $23 \mathrm{O} \mathrm{O} 23$ & -1.035651936 & 3.506463276 & 3.218357886 \\
\hline $24 \mathrm{H} \mathrm{H} 24$ & -1.462587124 & 3.014178486 & 3.939464685 \\
\hline
\end{tabular}




\begin{tabular}{|c|c|c|c|}
\hline \multicolumn{4}{|c|}{$\begin{array}{l}\text { TS 8a, 8b to } 9 \\
\text { E(B3LYP/6-31G*)=-1373.36481 au } \\
v=-385.45 \mathrm{~cm}^{-1} \\
\text { Cartesian Coordinates (Angstroms) }\end{array}$} \\
\hline Atom & $\mathrm{Y}$ & & \multirow[b]{2}{*}{2.494192920} \\
\hline $1 \mathrm{C} \mathrm{C} 1$ & -0.715719142 & -1.863718848 & \\
\hline $2 \mathrm{H} \mathrm{H} 2$ & -0.517168757 & -1.900219149 & 4.637787609 \\
\hline $3 \mathrm{C} \mathrm{C} 3$ & -0.044386732 & -1.742931287 & 3.673973039 \\
\hline $4 \mathrm{OO} 4$ & 1.241107764 & -1.377978601 & 3.769255697 \\
\hline $5 \mathrm{C} \mathrm{C5}$ & 2.083452612 & -1.525384876 & 2.575398200 \\
\hline $6 \mathrm{H} \mathrm{H6}$ & 2.901133631 & -0.824308908 & 2.765028566 \\
\hline $7 \mathrm{C} \mathrm{C7}$ & -0.028602387 & -1.537332793 & 1.262060157 \\
\hline $8 \mathrm{C} \mathrm{C} 8$ & 1.334209842 & & 1.301206131 \\
\hline 9 H H9 & 1.895320981 & -1.349887 & 0.386524057 \\
\hline $10 \mathrm{C} \mathrm{C} 10$ & -1.049485628 & 1.554410393 & 1.157844197 \\
\hline 11 H H11 & $-2.1265 t$ & 1.642019 & 1.243613364 \\
\hline $12 \mathrm{C} \mathrm{C} 12$ & 1.584151252 & 0.817781404 & 1.09 \\
\hline $13 \mathrm{C} \mathrm{C} 13$ & -0.416820181 & 1.533197698 & -0.065543863 \\
\hline $14 \mathrm{C} \mathrm{C} 14$ & 0.963357997 & 1.169431163 & -0.115448165 \\
\hline 150015 & -0.435466262 & 1.486114810 & 2.330746549 \\
\hline $16 \mathrm{C} \mathrm{C} 16$ & 1.032666478 & 1.493318496 & 2.341532870 \\
\hline 17 H H17 & 1.279542472 & 0.928525715 & 3.241698084 \\
\hline 18 H H18 & 2.668809318 & 0.738284043 & 1.064166586 \\
\hline 19 C C19 & 2.641358631 & -2.948152278 & 2.542555782 \\
\hline 20 H H20 & 3.364533295 & -3.046549883 & 1.726124573 \\
\hline $21 \mathrm{H} \mathrm{H} 21$ & 1.841364665 & & 2.387698853 \\
\hline $22 \mathrm{H} \mathrm{H} 22$ & 3.14699 & & 3.48 \\
\hline $23 \mathrm{C} \mathrm{C} 23$ & 1.505502744 & 2.93 & 2.49 \\
\hline $24 \mathrm{H} \mathrm{H} 24$ & 2.595799984 & 2.9 & 36016 \\
\hline $\mathrm{H} 25$ & 1.059 & 3.3 & 3.3 \\
\hline 26 H H26 & 1.229501695 & 3.541617880 & 1.628820353 \\
\hline $27 \mathrm{C} \mathrm{C} 27$ & -1.230746161 & 1.684576241 & -1.293400559 \\
\hline 28 C C 28 & 1.653700774 & 0.902489995 & -1.390110725 \\
\hline 29 C C29 & -0.466892338 & 1.759701594 & -2.590957538 \\
\hline 30 H H30 & -1.083454505 & 1.695461464 & -3.485789760 \\
\hline 31 C C31 & 0.960093451 & 1.366875727 & -2.642163298 \\
\hline 32 Н H32 & 1.393683940 & 1.010400033 & -3.574466490 \\
\hline 33 O O33 & 0.572236767 & 2.7495 & -2.621424587 \\
\hline $34 \mathrm{O} \mathrm{O} 34$ & -2.449646 & 1.7 & -1.29 \\
\hline $35 \mathrm{O} O 35$ & 2.747012629 & 0.349904268 & -1.437078041 \\
\hline 36 C C36 & -2.110549967 & -2.347841983 & 2.515125204 \\
\hline 37 C C37 & -0.701844492 & -1.569014303 & -0.022383340 \\
\hline $38 \mathrm{C} \mathrm{C} 38$ & -2.889489568 & -2.197894335 & 1.232278816 \\
\hline 39 Н H39 & -3.821956752 & -2.759102683 & 1.194681849 \\
\hline $40 \mathrm{C} \mathrm{C} 40$ & -2.194887181 & -1.800928293 & -0.018722282 \\
\hline $41 \mathrm{H} \mathrm{H} 41$ & -2.623131553 & -2.067890892 & -0.983216951 \\
\hline $42 \mathrm{O} 042$ & -2.959352045 & -0.852676606 & 0.739657628 \\
\hline $43 \mathrm{O} \mathrm{O} 43$ & -0.101055609 & -1.376093831 & -1.088711614 \\
\hline $44 \mathrm{O} O 44$ & -2.639699873 & -2.849000701 & 3.497581622 \\
\hline
\end{tabular}




\begin{tabular}{|c|c|c|c|}
\hline \multirow{2}{*}{\multicolumn{4}{|c|}{$\begin{array}{l}\text { TS 8a to homo-dimer } \\
\text { E(B3LYP/6-31G*)=-1373.36240 au } \\
\text { v=-393.30 } \mathrm{cm}^{-1} \\
\text { Cartesian Coordinates (Angstroms) } \\
\text { Atom X Y Z Z }\end{array}$}} \\
\hline & & & \\
\hline $\mathrm{C} \mathrm{C} 1$ & -0.071031958 & -1.484670315 & 1.3148703 \\
\hline $2 \mathrm{C} \mathrm{C} 2$ & 1.296532387 & -1.137257466 & 1.268597398 \\
\hline $3 \mathrm{C} \mathrm{C} 3$ & -0.957107570 & 1.903711082 & 1.233513439 \\
\hline $4 \mathrm{H} \mathrm{H} 4$ & -1.998462076 & 2.174802003 & 1.377771375 \\
\hline 5 C C5 & 1.578267280 & 0.836460612 & 1.016127527 \\
\hline 6 C C6 & -0.397319797 & 1.823703118 & -0.017814003 \\
\hline $7 \mathrm{C} \mathrm{C7}$ & 0.943553548 & 1.324388370 & -0.146416926 \\
\hline $80 \mathrm{O} 8$ & -0.309079850 & 1.707461 & 2.371542868 \\
\hline 9 C C9 & 1.140213448 & & 2.330168900 \\
\hline $10 \mathrm{H} \mathrm{H} 10$ & 1.2984834 & & 3.171176517 \\
\hline 11 H H11 & 2.6579407 & & 0.935197578 \\
\hline $12 \mathrm{C} \mathrm{C} 12$ & -0.712734690 & -1.84455 & 43958 \\
\hline $13 \mathrm{C} \mathrm{C} 13$ & 0.047734993 & -1.9 & -1.058522490 \\
\hline 14 H H14 & -0.400531796 & -2.103597 & -2.033383048 \\
\hline 150015 & 1.370178373 & -1.880193133 & -1.098049472 \\
\hline $16 \mathrm{C} \mathrm{C} 16$ & 2.113085995 & -1.804690159 & 0.166256936 \\
\hline 17 H H17 & 2.982060617 & -1.203992453 & -0.102168944 \\
\hline 18 H H18 & 1.801638131 & -1.135653477 & 2.232994963 \\
\hline 19 C C19 & 1.836805117 & 2.811591697 & 2.580732780 \\
\hline 20 H H20 & 2.917358622 & 2.656625758 & 2.677356743 \\
\hline 21 H H21 & 1.466594317 & 3.254974447 & 3.510535346 \\
\hline $22 \mathrm{H} \mathrm{H} 22$ & 1.660329245 & 3.511081351 & 1.757524190 \\
\hline 23 C C23 & 2.5406687 & -3.22 & 4522763 \\
\hline $24 \mathrm{H} \mathrm{H} 24$ & 3.200875518 & -3.19 & 1.418702317 \\
\hline 5 H H25 & 3.087631534 & -3.67 & -0.286178869 \\
\hline $6 \mathrm{H} \mathrm{H} 26$ & 1.67460 & -3.8 & 0.78 \\
\hline $27 \mathrm{C} \mathrm{C} 27$ & -1.220454409 & 2.198723979 & -1.188348447 \\
\hline $28 \mathrm{C} \mathrm{C} 28$ & -0.463854756 & 2.435819881 & -2.471166841 \\
\hline 29 H H29 & -1.082611474 & 2.557049781 & -3.358603444 \\
\hline 30 C C30 & 1.603795255 & 1.246453404 & -1.453290331 \\
\hline 31 C C31 & 0.942260557 & 1.975253061 & -2.595588790 \\
\hline 32 H H32 & 1.358984375 & 1.758128192 & -3.577729342 \\
\hline $33 \mathrm{O} \mathrm{O} 33$ & 0.632948715 & 3.352255436 & -2.347318891 \\
\hline $34 \mathrm{O} \mathrm{O} 34$ & 2.652198769 & $0.633290^{\circ}$ & -1.645657230 \\
\hline 35 C C35 & -2 & -2 & -0.021154009 \\
\hline 36 C C36 & -0.827973618 & -1.399082339 & 2.567530820 \\
\hline 37 C C37 & -2.890283651 & -2.304380940 & 1.282462417 \\
\hline 38 C C38 & -2.215354954 & -1.989505068 & 2.566964812 \\
\hline 39 H H39 & -3.977097107 & -2.310284915 & 1.219708428 \\
\hline $0 \mathrm{H} \mathrm{H} 40$ & -2.807797657 & -1.763670157 & 3.452101399 \\
\hline 10041 & -2.308028928 & -3.337607447 & 2.089903015 \\
\hline $42 \mathrm{O} \mathrm{O} 42$ & -0.383030801 & -0.886924571 & 3.593155328 \\
\hline $43 \mathrm{O} \mathrm{O} 43$ & -2.432609841 & 2.351257873 & -1.148873483 \\
\hline 44 O O44 & -2.786482672 & -2.083511033 & -1.077990488 \\
\hline
\end{tabular}




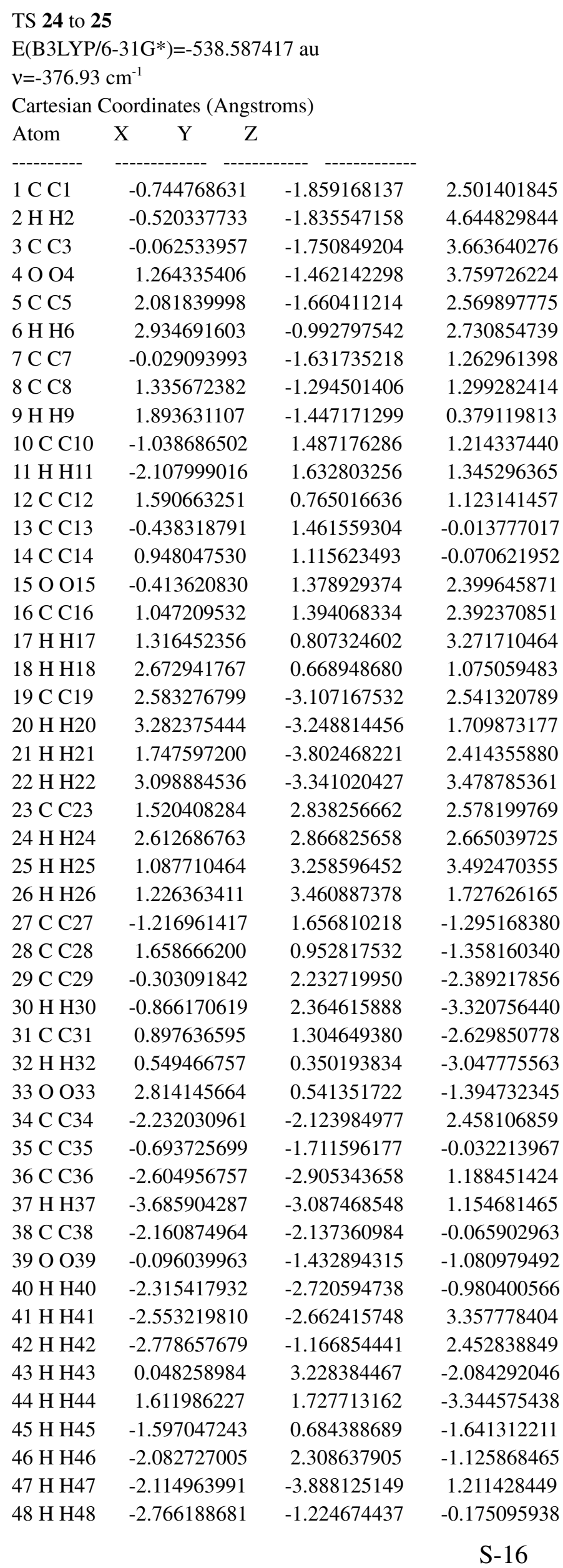




\begin{tabular}{|c|c|c|c|}
\hline $\begin{array}{l}\text { TS } 24 \text { to ho } \\
\text { E(B3LYP/C } \\
\text { v=-374.98 } \\
\text { Cartesian }\end{array}$ & $\begin{array}{l}\text { omo-dimer } \\
\left.6-31 \mathrm{G}^{*}\right)=-538.580 \\
\mathrm{~cm}^{-1} \\
\text { Coordinates (Angs }\end{array}$ & $\begin{array}{l}59 \text { au } \\
\text { oms) }\end{array}$ & \\
\hline Atom & $\mathrm{X}$ & & \\
\hline $\mathrm{C} \mathrm{C} 1$ & -0.058508984 & -1.449973344 & 1.227288412 \\
\hline $\mathrm{C} \mathrm{C} 2$ & 1.317833402 & -1.188752420 & 1.231118437 \\
\hline $\mathrm{C} \mathrm{C} 3$ & -0.951878560 & 1.831284161 & 1.280413391 \\
\hline $4 \mathrm{H} \mathrm{H} 4$ & -1.987189071 & 2.091838004 & 1.485040316 \\
\hline $5 \mathrm{C} \mathrm{C} 5$ & 1.610289262 & 0.882529597 & 1.000789544 \\
\hline $6 \mathrm{C} \mathrm{C} 6$ & -0.449138767 & 1.735027182 & 0.018001014 \\
\hline $7 \mathrm{C} \mathrm{C7}$ & 0.915396569 & 1.308831360 & -0.138630933 \\
\hline 8008 & -0.259180893 & 1.667175208 & 2.421604864 \\
\hline $9 \mathrm{CC} 9$ & 1.182834422 & 1.468203333 & 2.338009927 \\
\hline 10 H H10 & 1.391530348 & 0.778197642 & 3.157851523 \\
\hline 11 H H11 & 2.685420713 & 0.760311618 & 0.889064562 \\
\hline $12 \mathrm{C} \mathrm{C} 12$ & -0.694687690 & -1.720732206 & -0.033862989 \\
\hline $13 \mathrm{C} \mathrm{C} 13$ & 0.092799971 & -1.8 & -1.138963479 \\
\hline 14 H H14 & -0.310344848 & -1.989350072 & -2.137485976 \\
\hline 150015 & 1.437925306 & -1.835185148 & -1.155599481 \\
\hline $16 \mathrm{C} \mathrm{C} 16$ & 2.148124974 & -1.8 & 3936 \\
\hline 17 H H17 & 3.033552556 & -1.20 & -0.098 \\
\hline 18 H H18 & 1.803199150 & -1.190098435 & 2.204624990 \\
\hline 19 C C19 & 1.867261106 & 2.811744682 & 2.602450787 \\
\hline 20 H H20 & 2.953748589 & 2.678277723 & 2.662272765 \\
\hline $21 \mathrm{H} \mathrm{H} 21$ & 1.514596280 & 3.228714462 & 3.551570324 \\
\hline 22 H H 22 & 1.651338210 & 3.524576 & 1.799894175 \\
\hline $23 \mathrm{C} \mathrm{C} 23$ & 2.563569771 & -3.240875479 & 0.463394795 \\
\hline $24 \mathrm{H} \mathrm{H} 24$ & 3.199317515 & -3.245816457 & 1.356492375 \\
\hline $25 \mathrm{H} \mathrm{H} 25$ & 3.127424534 & -3.676279298 & -0.368208818 \\
\hline $26 \mathrm{H} \mathrm{H} 26$ & 1.685903205 & -3.864735209 & 0.661841674 \\
\hline $27 \mathrm{C} \mathrm{C} 27$ & -1.2893013 & 2.007345053 & -1.207015430 \\
\hline $28 \mathrm{C} \mathrm{C} 28$ & -0.430511790 & 2.67 & -2.2 \\
\hline 29 H H29 & -1.0332 & 2.8 & -3.1 \\
\hline 30 C C30 & 1.563301255 & 1.27 & 1306 \\
\hline 31 C C31 & 0.762751653 & 1.782791174 & -2.660748735 \\
\hline 32 Н H32 & 0.406316812 & 0.891588578 & -3.198870520 \\
\hline 33 O O33 & 2.704885748 & 0.844792609 & -1.616206255 \\
\hline 34 C C34 & -2.200709980 & -1.810324148 & -0.104008946 \\
\hline 35 C C35 & -0.828352614 & -1.402970361 & 2.483058824 \\
\hline 36 C C36 & -2.743688710 & -2.502384845 & 1.157053448 \\
\hline 37 C C37 & -2.320109933 & -1.732711186 & 2.416546885 \\
\hline 38 H H38 & -3.837042212 & -2.576366806 & 1.113302470 \\
\hline 39 Н Н39 & -2.853839 & -0.771425658 & 2.460625847 \\
\hline $40 \mathrm{O} 040$ & -0.307352881 & -1.104611 & 3.555383309 \\
\hline 41 H H41 & -2.580796811 & -2.268246936 & 3.335982450 \\
\hline $42 \mathrm{H} \mathrm{H} 42$ & -2.634713756 & -0.798814642 & -0.161450950 \\
\hline 43 H H43 & -2.514430818 & -2.344085884 & -1.009049520 \\
\hline 44 H H44 & -0.068959965 & 3.644152274 & -1.919268092 \\
\hline $45 \mathrm{H} \mathrm{H} 45$ & 1.463744328 & 2.285910917 & -3.335717438 \\
\hline 46 H H46 & -1.684803205 & 1.060343494 & -1.608920228 \\
\hline 47 H H47 & -2.153871995 & 2.631769785 & -0.951937564 \\
\hline 48 H H48 & -2.354416901 & -3.528548320 & 1.199926416 \\
\hline
\end{tabular}


28

$\mathrm{E}\left(\mathrm{B} 3 \mathrm{LYP} / 6-31 \mathrm{G}^{*}\right)=-1375.77840 \mathrm{au}$

Cartesian Coordinates (Angstroms)

\begin{tabular}{|c|c|c|c|}
\hline Atom & $\mathrm{X}$ & & \\
\hline $1 \mathrm{C} \mathrm{C} 1$ & -0.696354862 & -2.078453001 & 1.935192510 \\
\hline $2 \mathrm{H} \mathrm{H} 2$ & -0.209903560 & -2.061897434 & 4.030444869 \\
\hline $3 \mathrm{C} \mathrm{C} 3$ & 0.122395571 & -1.964988456 & 2.999514991 \\
\hline $4 \mathrm{O} \mathrm{O} 4$ & 1.432559452 & -1.644143255 & 2.920796737 \\
\hline $5 \mathrm{C} \mathrm{C} 5$ & 2.113097266 & -1.888007014 & 1.653631716 \\
\hline 6 H H6 & 2.943930610 & -1.174388228 & 1.662899352 \\
\hline 7 C C7 & -0.126739056 & -1.752305922 & 0.619570115 \\
\hline $8 \mathrm{C} \mathrm{C} 8$ & 1.209369791 & -1.607256707 & 0.485783057 \\
\hline 9 H H9 & 1.653586313 & -1.293654246 & -0.455263091 \\
\hline 10 C C10 & -1.278264350 & 2.371715856 & -0.341582431 \\
\hline 11 H H11 & -2.354747372 & 2.519458423 & -0.354595105 \\
\hline 12 C C12 & 1.399908067 & 1.834162249 & -0.007410277 \\
\hline 13 C C13 & -0.547916586 & 2.115370187 & -1.447683119 \\
\hline 14 C C14 & 0.854811835 & 1.742796665 & -1.236443280 \\
\hline $15 \mathrm{O} \mathrm{O} 15$ & -0.798431201 & 2.382582611 & 0.921963497 \\
\hline 16 C C16 & 0.639564804 & 2.453828251 & 1.130951687 \\
\hline 17 H H17 & 0.802498190 & 1.880617692 & 2.053155853 \\
\hline 18 H H18 & 2.426991516 & 1.526550653 & 0.162521687 \\
\hline 19 C C19 & 2.673526296 & -3.314397055 & 1.637153197 \\
\hline $20 \mathrm{H} \mathrm{H} 20$ & 3.260176627 & -3.476108255 & 0.725119178 \\
\hline $21 \mathrm{H} \mathrm{H} 21$ & 1.863350382 & -4.050794688 & 1.664022741 \\
\hline $22 \mathrm{H} \mathrm{H} 22$ & 3.322450336 & -3.470141625 & 2.505101375 \\
\hline 23 C C23 & 1.039259124 & 3.913800870 & 1.368032825 \\
\hline $24 \mathrm{H} \mathrm{H} 24$ & 2.102326662 & 3.975983031 & 1.626960984 \\
\hline $25 \mathrm{H} \mathrm{H} 25$ & 0.454364284 & 4.332628961 & 2.190853529 \\
\hline 26 H H26 & 0.867134462 & 4.511405589 & 0.465871185 \\
\hline 27 C C27 & -1.019253323 & -1.455178064 & -0.528871982 \\
\hline 28 C C28 & -2.138685885 & -2.488962217 & 2.090609361 \\
\hline 29 H H29 & -2.491322294 & -2.216440825 & 3.099187226 \\
\hline 30 C C30 & -2.492473730 & -1.333791740 & -0.239832912 \\
\hline 31 H H31 & -3.130861274 & -1.278542168 & -1.116841817 \\
\hline 32 C C32 & -3.040364122 & -1.804109070 & 1.053904415 \\
\hline 33 H H33 & -4.087425590 & -2.125351365 & 1.069590544 \\
\hline $34 \mathrm{O} \mathrm{O} 34$ & -2.825135718 & -0.413411223 & 0.824058449 \\
\hline $35 \mathrm{O} \mathrm{O} 35$ & -2.348231457 & -3.918673056 & 2.014824864 \\
\hline 36 H H36 & -1.801105779 & -4.234518804 & 1.297577088 \\
\hline 37 O O37 & -0.599702641 & -1.310364015 & -1.675138898 \\
\hline 38 C C38 & -1.211665943 & 1.989126812 & -2.806188430 \\
\hline 39 H H39 & -1.907851628 & 2.823182526 & -2.952897990 \\
\hline 40 C C40 & 1.668647484 & 1.213186004 & -2.365482945 \\
\hline $41 \mathrm{C}$ C41 & -0.197119703 & 2.024945850 & -3.940512985 \\
\hline 42 H H42 & -0.628211893 & 1.864464829 & -4.928400240 \\
\hline 43 C C43 & 1.210307863 & 1.606514272 & -3.736717291 \\
\hline 44 H H44 & 1.790517741 & 1.189388597 & -4.555797772 \\
\hline $45 \mathrm{O} \mathrm{O} 45$ & 0.841121708 & 2.994622009 & -3.879457442 \\
\hline 460046 & -2.022209741 & 0.823136517 & -2.962270932 \\
\hline 47 H H47 & -1.501084539 & 0.043875970 & -2.685565866 \\
\hline $48 \mathrm{O} \mathrm{O} 48$ & 2.653377564 & 0.504264776 & -2.211516251 \\
\hline
\end{tabular}




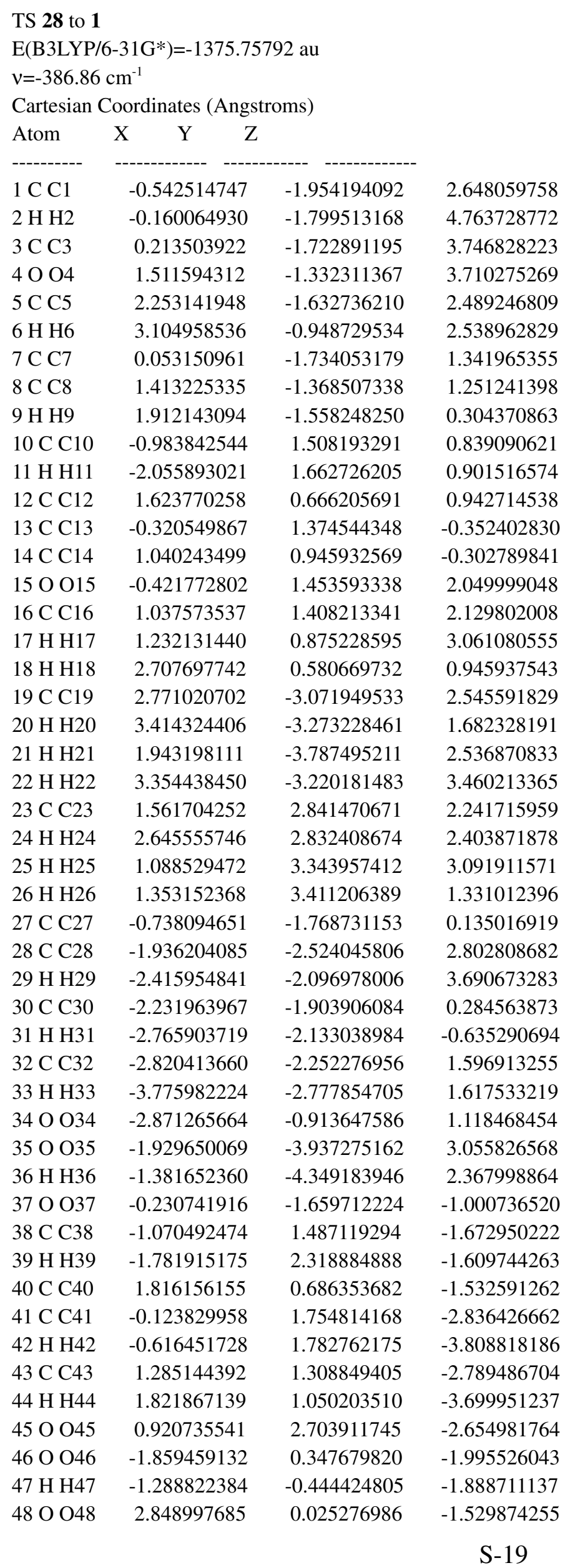


$\mathrm{E}\left(\mathrm{B} 3 \mathrm{LYP} / 6-31 \mathrm{G}^{*}\right)=-1375.78006 \mathrm{au}$

Cartesian Coordinates (Angstroms)

\begin{tabular}{|c|c|c|c|}
\hline Atom & $\mathrm{Y}$ & & \\
\hline $1 \mathrm{C} \mathrm{C} 1$ & -0.235325911 & -1.740339484 & 1.032970173 \\
\hline $2 \mathrm{C} \mathrm{C} 2$ & 1.102807778 & -1.693630142 & 0.893357564 \\
\hline $3 \mathrm{C} \mathrm{C} 3$ & -1.077610380 & 2.076115679 & 0.506397676 \\
\hline $4 \mathrm{H} \mathrm{H} 4$ & -2.158865044 & 2.141735108 & 0.548109842 \\
\hline $5 \mathrm{C} \mathrm{C} 5$ & 1.640593959 & 1.648514521 & 0.649939848 \\
\hline 6 C C6 & -0.374839353 & 2.081220332 & -0.644595478 \\
\hline 7 C C7 & 1.062551061 & 1.805314112 & -0.551813234 \\
\hline 8008 & -0.560126499 & 1.900429973 & 1.732285251 \\
\hline 9 C C9 & 0.889567228 & 1.913309771 & 1.926498185 \\
\hline 10 H H10 & 1.061938433 & 1.111442412 & 2.654587474 \\
\hline 11 H H11 & 2.697458961 & 1.402126535 & 0.721019696 \\
\hline $12 \mathrm{C} \mathrm{C} 12$ & -1.090273269 & -2.017267122 & -0.132881636 \\
\hline 13 C C13 & -0.483351383 & -2.173812707 & -1.321990987 \\
\hline 14 H H14 & -1.018819768 & -2.354137813 & -2.253576032 \\
\hline $15 \mathrm{O} \mathrm{O} 15$ & 0.850912171 & -2.048437023 & -1.542401356 \\
\hline 16 C C16 & 1.777366951 & -2.043836517 & -0.408198671 \\
\hline 17 H H17 & 2.516090846 & -1.281121747 & -0.678651828 \\
\hline 18 H H18 & 1.737028688 & -1.493655265 & 1.751725680 \\
\hline 19 C C19 & 1.286578303 & 3.263016350 & 2.541978291 \\
\hline $20 \mathrm{H} \mathrm{H} 20$ & 2.350549305 & 3.256548325 & 2.814783005 \\
\hline $21 \mathrm{H} \mathrm{H} 21$ & 0.697351461 & 3.448040125 & 3.447340976 \\
\hline $22 \mathrm{H} \mathrm{H} 22$ & 1.113299506 & 4.079260178 & 1.829470142 \\
\hline $23 \mathrm{C} \mathrm{C} 23$ & 2.458299955 & -3.418928352 & -0.327707568 \\
\hline $24 \mathrm{H} \mathrm{H} 24$ & 3.238628487 & -3.410367379 & 0.441694887 \\
\hline $25 \mathrm{H} \mathrm{H} 25$ & 2.920653331 & -3.664318575 & -1.290522153 \\
\hline $26 \mathrm{H} \mathrm{H} 26$ & 1.730926801 & -4.198597740 & -0.076059457 \\
\hline 27 C C27 & -1.070767646 & 2.063401249 & -1.975010592 \\
\hline $28 \mathrm{H} \mathrm{H} 28$ & -1.969176468 & 2.685866333 & -1.949281054 \\
\hline 29 C C29 & -0.166051880 & 2.526354287 & -3.103864621 \\
\hline 30 Н H30 & -0.623080782 & 2.524603505 & -4.093645801 \\
\hline 31 C C31 & 1.880460579 & 1.674053186 & -1.786038099 \\
\hline 32 C C32 & 1.297789553 & 2.301278481 & -3.020930679 \\
\hline 33 H H33 & 1.889299687 & 2.174605520 & -3.924180201 \\
\hline $34 \mathrm{O} \mathrm{O} 34$ & 0.711306335 & 3.606601980 & -2.839323800 \\
\hline $35 \mathrm{O} \mathrm{O} 35$ & 2.959672937 & 1.096431460 & -1.826568400 \\
\hline 36 C C36 & -2.590755234 & -1.891908213 & -0.023821485 \\
\hline 37 H H37 & -3.066619874 & -2.512275057 & -0.800863298 \\
\hline 38 C C38 & -0.858077809 & -1.571959861 & 2.376733195 \\
\hline 39 C C39 & -3.093685425 & -2.326042483 & 1.341958317 \\
\hline 40 C C40 & -2.244263840 & -2.132287288 & 2.533627613 \\
\hline $41 \mathrm{H} \mathrm{H} 41$ & -4.171241830 & -2.272747004 & 1.483382669 \\
\hline $42 \mathrm{H} \mathrm{H} 42$ & -2.692104483 & -1.981036754 & 3.513986744 \\
\hline $43 \mathrm{O} \mathrm{O} 43$ & -2.461268744 & -3.437569987 & 1.953195905 \\
\hline $44 \mathrm{O} \mathrm{O} 44$ & -0.294161345 & -1.037745678 & 3.319533671 \\
\hline $45 \mathrm{O} \mathrm{O} 45$ & -1.565743724 & 0.746432046 & -2.332881091 \\
\hline 46 H H46 & -0.814811875 & 0.132982551 & -2.262171772 \\
\hline $47 \mathrm{O} \mathrm{O} 47$ & -3.025703961 & -0.536383174 & -0.137140241 \\
\hline $48 \mathrm{H} \mathrm{H} 48$ & -2.632077925 & -0.157036609 & -0.947953866 \\
\hline
\end{tabular}




\begin{tabular}{|c|c|c|c|}
\hline \multicolumn{4}{|c|}{ 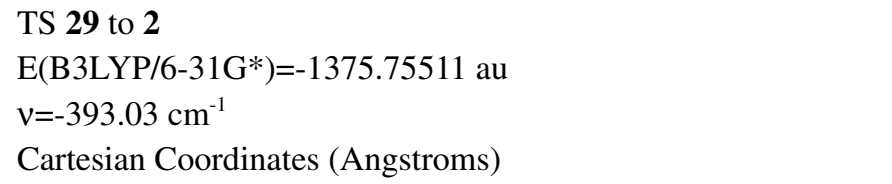 } \\
\hline Atom & $\begin{array}{llll}X & Y & Z\end{array}$ & & \\
\hline $1 \mathrm{C} \mathrm{C} 1$ & -0.160604743 & -1.412135283 & 1.319210955 \\
\hline $2 \mathrm{C} \mathrm{C} 2$ & 1.212355461 & -1.118988159 & 1.238715513 \\
\hline $3 \mathrm{C} \mathrm{C} 3$ & -0.996026633 & 1.886371910 & 1.041561891 \\
\hline $4 \mathrm{H} \mathrm{H} 4$ & -2.053346304 & 2.107096928 & 1.154031689 \\
\hline $5 \mathrm{C} \mathrm{C} 5$ & 1.559133510 & 0.871223836 & 0.985896757 \\
\hline $6 \mathrm{C} \mathrm{C6}$ & -0.380667814 & & -0.173101576 \\
\hline $7 \mathrm{C} \mathrm{C} 7$ & 0.980739292 & & -0.210619960 \\
\hline 8008 & -0.395065 & 1.74958 & 2.225617442 \\
\hline 9 C C9 & 1.048377638 & 1.523038188 & 2.265451558 \\
\hline 10 H H10 & 1.170573 & & 3.121945064 \\
\hline 11 H H11 & 2.640215 & & 0.965368870 \\
\hline 12 C C12 & -0.863109171 & -1.80 & 0.126342702 \\
\hline 13 C C13 & -0.136176980 & -2.023671645 & -1.004020328 \\
\hline 14 H H14 & -0.586350500 & -2.310 & -1.952760128 \\
\hline $15 \mathrm{O} 015$ & 1.203949590 & -1.959437812 & -1.099929532 \\
\hline $16 \mathrm{C} \mathrm{C} 16$ & 1.989582433 & -1.8 & 0.123563359 \\
\hline 17 H H17 & 2.840362733 & -1.211 & -0.198929435 \\
\hline 18 H H18 & 1.738973837 & -1.123375036 & 2.191975490 \\
\hline 19 C C19 & 1.735818037 & & 2.527631033 \\
\hline $20 \mathrm{H} \mathrm{H} 20$ & 2.811132583 & 2.711190945 & 2.677485122 \\
\hline 21 H H21 & 1.322094335 & 3.321 & 45028 \\
\hline 22 H H22 & 1.59 & & 6479 \\
\hline $23 \mathrm{C} \mathrm{C} 23$ & 2.4554 & -3.2 & 3424 \\
\hline $24 \mathrm{H} \mathrm{H} 24$ & 3.14814 & -3.1 & 9383 \\
\hline $25 \mathrm{H} \mathrm{H} 25$ & 2.975883322 & -3.69 & -0.267 \\
\hline $26 \mathrm{H} \mathrm{H} 26$ & 1.606649318 & -3.822 & 0.866747316 \\
\hline 27 C C27 & -1.144641813 & 2.005614380 & -1.455905100 \\
\hline $28 \mathrm{H} \mathrm{H} 28$ & -1.997658098 & 2.669027445 & -1.287748578 \\
\hline 29 C C29 & -0.251620065 & 2.569278585 & -2.552109332 \\
\hline 30 Н Н30 & -0.750135544 & 2.755751300 & -3.503853640 \\
\hline 31 C C31 & 1.744287250 & 1.342685565 & -1.463700989 \\
\hline 32 C C32 & 1.183175636 & 2.21 & -2.567734659 \\
\hline 33 Н H33 & 1.734034181 & 2.157405521 & -3.505282788 \\
\hline 34 O O34 & 0.733634218 & $3.51 \mathrm{c}$ & -2.17 \\
\hline $35 \mathrm{O} O 35$ & 2.789417435 & 0.71 & -1.641 \\
\hline 36 C C36 & -2.378264633 & -1.900272437 & 0.131166944 \\
\hline 37 Н H37 & -2.696199731 & -2.543646786 & -0.706057905 \\
\hline 38 C C38 & -0.850803160 & -1.416328588 & 2.617230541 \\
\hline 39 C C39 & -2.876235959 & -2.493063233 & 1.439823253 \\
\hline 40 C C40 & -2.133237637 & -2.201591854 & 2.680112750 \\
\hline $41 \mathrm{H} \mathrm{H} 41$ & -3.956773641 & -2.622129450 & 1.504023800 \\
\hline $42 \mathrm{H} \mathrm{H} 42$ & -2.644656198 & -2.160518511 & 3.639574893 \\
\hline $43 \mathrm{O} \mathrm{O} 43$ & -2.095552456 & -3.508211783 & 2.052716689 \\
\hline $44 \mathrm{OC}$ & -0.414626055 & -0.863324 & 3.624050957 \\
\hline $45 \mathrm{O} 045$ & & 0.792240094 & -1.984767558 \\
\hline 46 H H46 & -1.020915256 & 0.134059796 & -2.032640666 \\
\hline $47 \mathrm{O} O 47$ & -3.034101735 & -0.637541337 & 0.072507727 \\
\hline $48 \mathrm{H} \mathrm{H} 48$ & -2.709386981 & -0.152741596 & -0.714490605 \\
\hline
\end{tabular}

